\title{
Conectividade do grafo aleatório de Erdös-Rényi e uma variante com conexões locais
}

Elizbeth Chipa Bedia 

SERVIÇO DE PÓS-GRADUÇÃO DO ICMC-USP

Data de Depósito:

Assinatura:

\section{Elizbeth Chipa Bedia}

\section{Conectividade do grafo aleatório de Erdös-Rényi e uma variante com conexões locais}

Dissertação apresentada ao Instituto de Ciências Matemáticas e de Computação - ICMC-USP e ao Departamento de Estatística - DEs-UFSCar, como parte dos requisitos para obtenção do título de Mestre em Estatística - Interinstitucional de Pós-Graduação em Estatística. VERSÃO REVISADA.

Área de Concentração: Estatística

Orientador: Prof. Dr. Alexsandro Giacomo Grimbert Gallo

Co-orientador: Prof. Dr. Pablo Martin Rodriguez

\section{USP/UFSCar - São Carlos \\ Maio de 2016}


Ficha catalográfica elaborada pela Biblioteca Prof. Achille Bassi e Seção Técnica de Informática, ICMC/USP, com os dados fornecidos pelo(a) autor(a)

Chipa Bedia, Elizbeth
Conectividade do grafo aleatório de Erdös-Rényi e
uma variante com conexões locais / Elizbeth Chipa
Bedia; orientador Alexsandro Giacomo Grimbert
Gallo; co-orientador Pablo Martin Rodriguez. --
São Carlos, 2016.
$54 \mathrm{p.}$
Dissertação (Mestrado - Programa
Interinstitucional de Pós-graduação em Estatística) --
Instituto de Ciências Matemáticas e de Computação,
Universidade de São Paulo, 2016.
1. Conectividade. 2. Grafos aleatórios. 3.
Transição de fase. 4. Probabilidade. I. Grimbert
Gallo, Alexsandro Giacomo, orient. II. Martin
Rodriguez, Pablo, co-orient. III. Título.




\title{
Elizbeth Chipa Bedia
}

\section{Connectivity for the Erdös-Rényi random graph and a variant with local connections}

\author{
Master dissertation submitted to the Instituto de \\ Ciências Matemáticas e de Computação - ICMC- \\ USP and to the Departamento de Estatística - \\ DEs-UFSCar, in partial fulfillment of the require- \\ ments for the degree of the Master Joint Graduate \\ Program in Statistics DEs-UFSCar/ICMC-USP. FI- \\ NAL VERSION. \\ Concentration Area: Statistics \\ Advisor: Prof. Dr. Alexsandro Giacomo Grimbert \\ Gallo \\ Coadvisor: Prof. Dr. Pablo Martin Rodriguez
}

\section{USP/UFSCar - São Carlos}

May 2016 

Agradeço primeiramente a Deus pela vida e saúde. Ao meu orientador Prof. Dr. Alexsandro Giacomo Grimbert Gallo, e co-orientador Prof. Dr. Pablo Martin Rodriguez pelos ensinamentos, pela sua disponibilidade, apoio e confiança que gentilmente me mostraram na elaboração deste trabalho. Aos meus familiares que estiverem sempre presentes me apoiando e dando força para continuar nesta etapa da minha vida. A Capes, pelo auxílio financeiro. 



\section{Resumo}

Dizemos que um grafo é conectado se existe um caminho de arestas entre quaisquer par de vértices. O grafo aleatório de Erdös-Rényi com $n$ vértices é obtido conectando cada par de vértice com probabilidade $p_{n} \in(0,1)$, independentemente dos outros. Neste trabalho, estudamos em detalhe o limiar da conectividade na probabilidade de conexão $p_{n}$ para grafos aleatórios Erdös-Rényi quando o número de vértices $n$ diverge. Para este estudo, revisamos algumas ferramentas probabilísticas básicas (convergência de variáveis aleatórias e Métodos do primeiro e segundo momento), que também irão auxiliar ao melhor entendimento de resultados mais complexos. Além disto, aplicamos os conceitos anteriores para um modelo com uma topologia simples, mais especificamente estudamos o comportamento assintótico da probabilidade de não existência de vértices isolados, e discutimos a conectividade ou não do grafo. Por fim mostramos a convergência em distribuição do número de vértices isolados para uma Distribuição Poisson do modelo estudado.

Palavras-chave: Conectividade, Grafos aleatórios, Transição de fase, Probabilidade. 



\begin{abstract}
We say that a graph is connected if there is a path edges between any pair of vertices. Random graph Erdös-Rényi with $n$ vertices is obtained by connecting each pair of vertex with probability $p_{n} \in(0,1)$ independently of the others. In this work, we studied in detail the connectivity threshold in the connection probability $p_{n}$ for random graphs Erdös-Rényi when the number of vertices $n$ diverges. For this study, we review some basic probabilistic tools (convergence of random variables and methods of the first and second moment), which will lead to a better understanding of more complex results. In addition, we apply the above concepts for a model with a simple topology, specifically studied the asymptotic behavior of the probability of non-existence of isolated vertices, and we discussed the connectivity or not of the graph. Finally we show the convergence in distribution of the number of isolated vertices for a Poisson distribution of the studied model.
\end{abstract}

Key-words: Connectivity, Random graphs, Phase transition, Probability. 



\section{Lista de Figuras}

1.1 Simulação de $E R_{n}(\lambda / n)$, com $n=100$ e $\lambda=1 / 2$ e $3 / 2$ respectivamente. . 10

$1.2 E R_{n}(\lambda / n)$ para $\lambda=5$ variando $n \ldots \ldots \ldots \ldots \ldots \ldots$

$1.3 E R_{n}(\lambda / n)$ para $\lambda=\log \sqrt{n}$ variando $n \ldots \ldots \ldots . \ldots \ldots$

$1.4 E R_{n}(\lambda / n)$ para $\lambda=2 \log n$ variando $n \ldots \ldots \ldots \ldots \ldots$

$1.5 E R_{n}(\lambda / n)$ para $\lambda=\log n$ variando $\ldots \ldots \ldots \ldots \ldots$

3.1 Representação gráfica de $G_{n}(p, q, 1) \ldots \ldots \ldots \ldots \ldots$

$1 \quad$ Probabilidade de sobrevivência, para um processo de ramificação com média $\lambda .53$ 



\section{Sumário}

$\begin{array}{ll}\text { Introdução } & 6\end{array}$

1 O grafo aleatório de Erdös-Rényi 8

1.1 Descrição do modelo . . . . . . . . . . . . . . . . . . . 8

1.2 Conectividade . . . . . . . . . . . . . . . . . . . . . . . . . 9

1.3 Janela crítica para a conectividade . . . . . . . . . . . . . 15

2 Vértices isolados e conectividade de $E R_{n}(p) \quad 16$

2.1 Métodos Probabilísticos . . . . . . . . . . . . . . . . . 16

2.1.1 Convergência para uma variável aleatória Poisson . . . . . . . . . . 16

2.1.2 Métodos do primeiro e segundo momento . . . . . . . . . . . . . 23

2.2 Prova do Teorema de limiar da Conectividade para $E R_{n}(p) \ldots \ldots$. . . . 25

2.2.1 Controle dos momentos do número de vértices isolados . . . . . . . 25

2.2.2 Controlar $\mathbb{P}_{\lambda}(Y=0)$ basta para estudar a conectividade . . . . . . 27

2.2 .3 Prova do Teorema 1.2 .9 . . . . . . . . . . . . . . . . 30

2.3 Prova do Teorema da Janela crítica para a conectividade . . . . . . . . . 31

2.3.1 Prova do Teorema 1.3.1 . . . . . . . . . . . . . . . 32

3 Grafo aleatório com topologia simples 33

3.1 Descrição do modelo . . . . . . . . . . . . . . . . . . . . . 33

3.2 Esperança e variância de vértices isolados . . . . . . . . . . . . . . . . . . . . . . . . . . . . 38

3.3 Distribuição dos vértices isolados . . . . . . . . . . . . . . . . . . . . . . . . . . . . . . . . . . . . . .

3.4 Prova do Teorema 3.1.1 . . . . . . . . . . . . . . . . . . 41

4 Conclusão $\quad 43$

$\begin{array}{ll}\text { Apêndice } & 44\end{array}$

A1. Permutações e Combinações . . . . . . . . . . . . . . . . . . . 44

A1.1. Permutações simples . . . . . . . . . . . . . . . . . . . . . 44

A1.2. Permutações simples . . . . . . . . . . . . . . . . . . . . . . . 44

A1.3. Primeiro Lema de Kaplansky . . . . . . . . . . . . . . . . . . . . . . . . . . . . . . . . 45

A1.4. Segundo Lema de Kaplansky . . . . . . . . . . . . . . . . . . . . . . . . . . . . . . . 45

A2. Probabilidades . . . . . . . . . . . . . . . 46

A2.1. Propriedades . . . . . . . . . . . . . . . . 46

A3. Convergência de variáveis aleatórias . . . . . . . . . . . . . . 47

A4. Definição das funções $o$ e $O \ldots \ldots$. . . . . . . . . . . . . . . . 49 


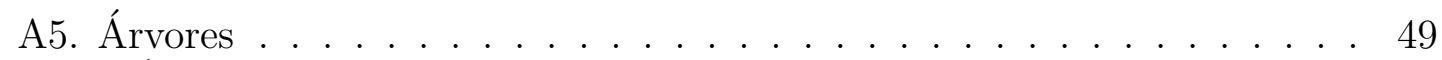

A5.1. Árvores . . . . . . . . . . . . . . . . . . . . . . . . . . . . . . . . . . 59

A5.2. Árvores de extensão . . . . . . . . . . . . . . . . . . . . . . 50

A6. Processos de Ramificação . . . . . . . . . . . . . . . . . . . . . . 50

A6.1. Processo de ramificação Poisson . . . . . . . . . . . . . 52 


\section{Introdução}

A teoria de grafos aleatórios é iniciada em 1959-1960 com os artigos "On random graphs I" e "On the evolution of random graphs" de Erdös e Rényi (ver [8, 9]), e desde então tem sido desenvolvida com o objetivo de responder perguntas de interesse tanto físico quanto matemático. Nas últimas décadas nota-se um grande interesse no estudo de grafos aleatórios e processos em grafos aleatórios, devido principalmente ao fato de que esta classe de estruturas pode ser usada para representar estruturas reais, como por exemplo, a internet, sistemas de redes elétricas de uma cidade, relações sociais, etc. Ver por exemplo [7].

O modelo original proposto por Erdös e Rényi pode ser descrito como um modelo estocástico a tempo discreto. O modelo começa no tempo 0 com $n$ vértices sem arestas entre eles, e novos elos são adicionados ou não, um a cada vez, uniformemente ao acaso entre todos os elos que ainda não estão presentes e que não foram testados em instantes anteriores. Em outras palavras, no modelo de Erdös-Rényi com probabilidade de conexão $p$, que denotaremos por $E R_{n}(p), n$ vértices são considerados e cada par de vértices tem uma probabilidade $p$, independente dos demais pares, de estar conectado entre si por uma aresta.

Uma questão de interesse é a analise da conectividade do grafo resultante, fenômeno que está intimamente ligado com a não existência de vértices isolados. Sabe-se que quando existe pelo menos um vértice isolado então o grafo é desconectado, mas a recíproca não é verdadeira em geral, veremos esta situação com mais detalhe no Capítulo 2.2. No caso do grafo aleatório de Erdös e Rényi pode-se mostrar que quando não há vértices isolados, o grafo aleatório é conectado com alta probabilidade (i.e., com probabilidade tendendo para 1 quando $n \rightarrow \infty$ e denotado por c.a.p). Esta propriedade pode ser consultada em $[1,2]$.

O propósito do presente trabalho é descrever as ferramentas necessárias da teoria de probabilidades para estudar a propriedade de conectividade no caso particular do grafo aleatório de "Erdös-Rényi". Usaremos esta abordagem como uma primeira tentativa de estudo do problema de conectividade em um modelo simples de grafo aleatório descrito como segue. Considere $n$ vértices que se localizam num anel, cada vértice tem dois vizinhos, um à direita e um à esquerda. Com estrutura de vizinhança, cada vértice deste grafo é conectado com seus vértices vizinhos com probabilidade $q$, de forma independente. Além disso, qualquer par de vértices do grafo é conectado com probabilidade $p$, independentemente dos outros pares de vértices. 
A motivação deste trabalho segue do fato de que o modelo de Erdös-Rényi é considerado um dos mais simples e não representa muito bem os grafos do mundo real, que são mais complexos. Por isto, seria interessante estudar um modelo que se assemelha mais às redes do mundo real. Neste estudo complicamos um pouco mais o modelo Erdös-Rényi, adicionando uma topologia simples para estudar a conectividade e em particular os vértices isolados. Apesar do nosso novo modelo não ser de muita utilidade para modelar redes do mundo real, poderia ser considerado como uma iniciativa para estudos de modelos mais complicados considerando probabilidades de conexão que dependem da distância entre os vértices no anel. Alem disso, a partir de um ponto de vista matemático, este modelo é interessante pois inclui algo desejável, a topologia.

A dissertação está organizada da seguinte forma. No Capítulo 1, descrevemos formalmente o modelo Erdös-Rényi, logo definimos alguns conceitos concernente ao tema deste trabalho. Também apresentamos o teorema de limiar de conectividade e o teorema da janela crítica da conectividade, que são resultados principais deste trabalho, explicando eles com gráficos ilustrativos. No Capítulo 2, revisamos os métodos probabilísticos necessários para a prova dos teoremas relacionados à conectividade apresentados no Capítulo 1. As provas de tais teoremas são desenvolvidas no mesmo capítulo. No Capítulo 3, apresentamos um modelo de grafo aleatório com topologia simples, encontraremos a variância e esperança do número de vértices isolados e discutiremos o comportamento destes valores em relação a conectividade. Por fim provaremos que o número de vértices isolados para o novo modelo, converge para uma v.a. Poisson. 


\section{Capítulo 1}

\section{O grafo aleatório de Erdös-Rényi}

Neste capítulo apresentamos formalmente a descrição do modelo de Erdös-Rényi, explicando a transição de fase para este modelo. Assim também apresentamos o teorema de limiar da conectividade cujo entendimento e demostração é um dos objetivos principais deste trabalho. Por fim veremos a janela crítica para a conectividade do grafo ErdösRényi.

\subsection{Descrição do modelo}

Um conjunto finito não vazio de pontos $V=\left\{v_{1}, \ldots, v_{n}\right\}$ e o conjunto $E$ de diferentes pares não ordenados $\left(v_{i}, v_{j}\right)$ com $v_{i}, v_{j} \in V$ e $i \neq j$ é chamado um grafo $G$; que pode ser escrito como $G=\{V, E\}$. O número $n$ é chamado de tamanho do grafo. Os pontos $\left\{v_{1}, \ldots, v_{n}\right\}$ são chamados vértices e os pares $\left(v_{i}, v_{j}\right)$ as arestas do grafo.

Num grafo aleatório Erdös-Rényi, cada par de vértices $\left\{v_{i}, v_{j}\right\}$ é conectado com probabilidade $p \in(0,1)$, e de forma independente dos outros pares de vértices.

Uma variável aleatória muito importante no estudo de grafos aleatórios é o número de vizinhos que tem cada vértice do grafo ou grau, cuja distribuição é preciso conhecer. Para isto considere a v.a $Z_{i j}$ que indica se os vértices $v_{i}$ e $v_{j}$ estão conectados ou não, isto é

$$
Z_{i j}=\left\{\begin{array}{l}
1, v_{i} \text { e } v_{j} \text { estão conectados } \\
0, \text { caso contrário. }
\end{array}\right.
$$

Como $\mathbb{P}\left(Z_{i j}=1\right)=p$ e $\mathbb{P}\left(Z_{i j}=0\right)=1-p$, então $Z_{i j}$ segue uma distribuição $\operatorname{Bernoulli}(p)$. Note que um vértice é conectado com igual probabilidade $p$ com cada um dos outros $n-1$ vértices do grafo, de forma independente, logo a probabilidade de que um vértice tenha exatamente $k$ arestas é dada por uma distribuição $\operatorname{Binomial}(n-1, p)$, isto é,

$$
\mathbb{P}\left[X^{*}=k\right]=\left(\begin{array}{c}
n-1 \\
k
\end{array}\right) p^{k}(1-p)^{n-1-k},
$$

onde a v.a. $X^{*}$ é o número de arestas de um vértice. O grau médio do grafo é dado por 
$(n-1) p$. Se $p=\lambda /(n-1)$, então podemos escrever esta distribuição como

$$
\mathbb{P}\left[X^{*}=k\right]=\left(\begin{array}{c}
n-1 \\
k
\end{array}\right)\left(\frac{\lambda}{n-1}\right)^{k}\left(1-\frac{\lambda}{n-1}\right)^{n-1-k} \approx \frac{e^{-\lambda} \lambda^{k}}{k !},
$$

Ou seja, quando $n$ é suficientemente grande e $p=\lambda /(n-1)$, a distribuição de grau de um vértice é aproximadamente uma Poisson com média $\lambda$. Veremos isto com mais detalhe no Capítulo 2.1. No presente trabalho $\lambda$ poderá depender de $n$, mas por facilidade de notação vamos escrever só $\lambda$.

Um fenômeno muito importante no estudo de grafos aleatórios e que esta relacionado com a conectividade do grafo, é a transição de fase para a maior componente conectada, vamos entender melhor esta propriedade na Seção 1.2

\subsection{Conectividade}

Nesta seção veremos definições básicas, para entender melhor questões de interesse para este trabalho como a transição de fase e o limiar da conectividade do grafo.

Definição 1.2.1 (Caminho). Um caminho entre os vértices $v_{1}$ e $v_{j}$ é uma sequência de vértices $\left\{v_{1}, v_{2}, v_{3}, \ldots, v_{j-1}, v_{j}\right\}$ tal que $\left(v_{1}, v_{2}\right),\left(v_{2}, v_{3}\right), \ldots,\left(v_{j-1}, v_{j}\right)$ são arestas do grafo.

Definição 1.2.2 (Vértices conectados). Dizemos que os vértices $v_{i}, v_{j} \in V$ são conectados se existe um caminho entre $v_{i}$ e $v_{j}$, caso contrário dizemos que eles são desconectados. Se além disso esses vértices forem conectados por um caminho de tamanho 1, ou seja, por uma única aresta, eles são chamados de vértices adjacentes ou vizinhos.

Definimos uma notação. Para dois vértices $v_{i}, v_{j} \in V$ escrevemos $v_{i} \leftrightarrow v_{j}$ se estão conectados. Caso contrário escrevemos $v_{i} \leftrightarrow v_{j}$. Por convenção assumimos que $v_{i} \leftrightarrow v_{i}$.

Definição 1.2.3 (Componente conectada de um vértice). A componente conectada de um vértice $v_{i}$ é o conjunto $\mathcal{C}\left(v_{i}\right)$ de vértices aos quais está conectado, isto é

$$
\mathcal{C}\left(v_{i}\right):=\left\{x \in V: v_{i} \leftrightarrow x\right\}
$$

Denotamos o tamanho da componente conectada do vértice $v_{i}$ por $\left|\mathcal{C}\left(v_{i}\right)\right|$, é dizer, $\left|\mathcal{C}\left(v_{i}\right)\right|$ denota o número de vértices conectados com $v_{i}$.

Definição 1.2.4 (Maior componente conectada). Seja $G=\{V, E\}$ um grafo aleatório. Dizemos que $\mathcal{C}_{\max }(G)$ é maior componente conectada do grafo, que pode ser alguma componente conectada $\mathcal{C}(v)$ de um vértice $v \in V$, se satisfaz

$$
\left|\mathcal{C}_{\max }(G)\right|=\max _{v \in V}|\mathcal{C}(v)| .
$$

Num grafo podem existir mais de uma componentes conectada além da maior. Denotemos por $\mathcal{C}_{(2)}(G)$ a segunda maior componente conectada, cujo tamanho está definido como

$$
\left|\mathcal{C}_{(2)}(G)\right|:=\max _{v \in V^{\prime}}|\mathcal{C}(v)|
$$

onde $V^{\prime}$ é o conjunto dos vértices que não pertencem à maior componente conectada $\mathcal{C}_{\max }(G)$. 
Definição 1.2.5 (sequência de grafos altamente conectados). Uma sequência de grafos $\left(G_{n}\right)_{n \geq 1}$ é chamado altamente conectada, quando

$$
\liminf _{n \rightarrow \infty} \frac{\left|\mathcal{C}_{\max }\left(G_{n}\right)\right|}{n}>0
$$

Nesse caso $\mathcal{C}_{\text {max }}\left(G_{n}\right)$ é chamado componente gigante. Além disso, para uma sequência de grafos altamente conectados, esta componente gigante é chamada única quando

$$
\liminf _{n \rightarrow \infty} \frac{\left|\mathcal{C}_{(2)}\left(G_{n}\right)\right|}{n}=0
$$

Por simplicidade, no que segue do trabalho denotaremos $\mathcal{C}_{\max }\left(G_{n}\right)$ por $\mathcal{C}_{\max }$.

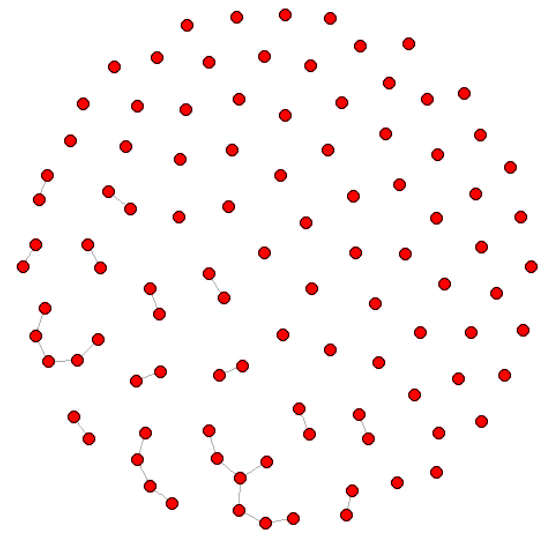

(a)

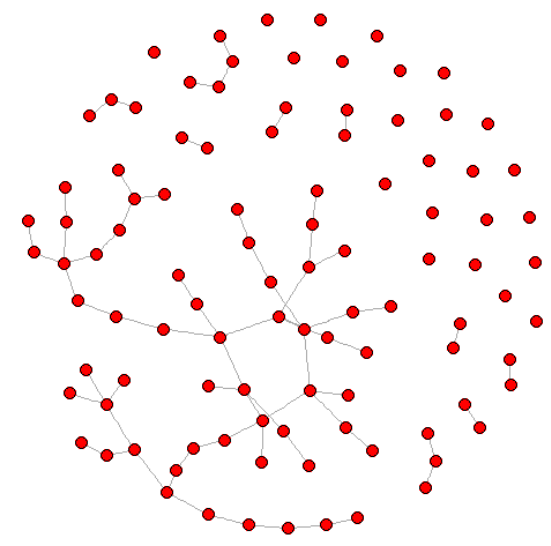

(b)

Figura 1.1: Simulação de $E R_{n}(\lambda / n)$, com $n=100$ e $\lambda=1 / 2$ e 3/2 respectivamente.

Uma transição de fase ocorre quando há uma transição repentina no tamanho da maior componente conectada. No grafo aleatório $E R_{n}(\lambda / n)$, a transição de fase acontece de acordo com o número médio de arestas por vértice $\lambda$, isto é, quando $\lambda<1$, então todas as componentes conectadas do grafo são muito pequenas, enquanto que quando o número médio de arestas $\lambda>1$, então existe uma única componente gigante que contem uma proporção positiva de vértices. Por exemplo na figura 1.1, mostramos dois grafos aleatórios Erdös-Rényi de tamanho $n=100$ e grau médio $\lambda=1 / 2$ e $3 / 2$ respectivamente. No grafo (a) vemos que $\lambda<1$, que também é conhecido como regime subcrítico ou fase subcrítica, neste caso os tamanhos das componentes conectadas são muito pequenas. Mas no grafo (b) temos que $\lambda>1$, que é conhecido como regime supercrítico, e podemos observar que existe um única componente conectada e cujo tamanho é bastante grande. 
E bem conhecido que a fase de transição da maior componente conectada para o modelo Erdös-Rényi ocorre da seguinte forma: Considere o grafo $E R_{n}(\lambda / n)$, com $p=\lambda / n$ :

a. Se $\lambda<1$, então o tamanho da maior componente conectada $\left|\mathcal{C}_{\text {max }}\right|$ é da ordem $\log n$. Isto é, $\left|\mathcal{C}_{\text {max }}\right|$ é muito pequena em relação ao tamanho do grafo, então o modelo não exibe componente gigante da ordem proporcional a $n$.

b. Enquanto se $\lambda>1$, então o tamanho da única componente gigante $\left|\mathcal{C}_{\text {max }}\right|$ é da ordem $\zeta(\lambda) n$, em que $\zeta(\lambda)<1$ e é a solução da equação $e^{-\lambda \zeta}=1-\zeta$. Ver [12, cap.1, pag. 5].

A Transição de fase da maior componente conectada é uma propriedade muito estudada, por exemplo podemos encontrar ela em [1, Cap.4].

Dizemos que $g(n)=O(f(n))$ quando $n \rightarrow \infty$, quando $f(n)>0$ e $\lim _{\sup } \rightarrow \infty \frac{|g(n)|}{f(n)}<\infty$. Ver Apêndice A3.

Teorema 1.2.6 (Lei dos grandes números para a componente gigante). Fixe $\lambda>1$. Então para todo $\nu \in\left(\frac{1}{2}, 1\right)$, existe $\delta=\delta(\nu, \lambda)>0$ tal que

$$
\mathbb{P}_{\lambda}\left(|| \mathcal{C}_{\text {max }}\left|-\zeta_{\lambda} n\right| \geq n^{\nu}\right)=O\left(n^{-\delta}\right)
$$

em que $\left|\mathcal{C}_{\text {max }}\right|$ representa o tamanho da componente maior.

Fazendo uma análise intuitiva deste teorema podemos dizer que um vértice tem maior componente conectada com probabilidade $\zeta_{\lambda}$, portanto a quantidade de vértices pertencentes à maior componente conectada é da ordem de $\zeta_{\lambda} n$. O Teorema 1.2.6 implica que quase todos estes vértices em componentes grandes estão, de fato, na mesma componente conectada, que é a componente gigante. Não vamos entrar em detalhe na prova deste teorema, mas podemos encontrar ele em [1, Cap.4, pág. 133]. Este teorema vai nos ajudar a entender melhor a importância da limiar da conectividade que apresentamos no Teorema 1.2.9. Mas antes precisamos de algumas definições.

Definição 1.2.7 (Grafo conectado). Dizemos que um grafo é conectado se todos seus vértices pertencem à mesma componente conectada. Caso contrário dizemos que é desconectado.

No Teorema 1.2.6 podemos ver que para todo $\lambda>1$, a maior componente conectada de $E R_{n}(\lambda / n)$ tem tamanho de ordem

$$
\zeta_{\lambda} n(1+o(1))
$$

quando $p=\lambda / n$, onde usando uma comparação com o processo de ramificação com distribuição de descendência dada por uma v.a. Poisson $(\lambda), \zeta_{\lambda}>0$, representa a probabilidade de sobrevivência do processo de ramificação, ver Apêndice A4. Por outro lado, para o processo de ramificação Poisson $(\lambda)$, podemos escrever

$$
\left.\zeta_{\lambda} \leq 1-\mathbb{P} \text { (a raiz não tenha descendentes }\right)=1-e^{-\lambda}<1,
$$


desde que a extinção do processo ocorre quando a raiz não tem descendentes. Então $\zeta_{\lambda} n<n$. Note que um grafo é conectado se o tamanho da maior componente é igual a $n$. Portanto, $E R_{n}(\lambda / n)$ é desconectado com alta probabilidade para todo $\lambda<\infty$ fixo. Se queremos ter conexão c.a.p, precisamos levar $\lambda$ para o infinito com $n$. O seguinte teorema mostra qual é o comportamento de $\lambda_{n}$ para garantir a conectividade c.a.p. Para entender melhor esta parte pode ver [1, cap.5, pag. 126].

Definição 1.2.8. Seja $G_{n}$ um grafo aleatório. Dizemos que uma sequência de grafos aleatórios $\left(G_{n}\right)_{n \geq 1}$ é conectada com alta probabilidade se $\mathbb{P}_{\lambda}\left(G_{n}\right.$ conectado $) \rightarrow 1$ quando $n \rightarrow \infty$.

Dada esta definição, veja o seguinte teorema

Teorema 1.2.9 (Limiar de Conectividade). Seja $E R_{n}(p)=E R_{n}$, com $p=\lambda / n$. Para $\lambda-\log n \rightarrow \infty$, a sequência de grafos $\left(E R_{n}\right)_{n \geq 1}$ é conectada com alta probabilidade, enquanto para $\lambda-\log n \rightarrow-\infty$, é desconectada com alta probabilidade.

A seguir, apresentamos exemplos gráficos para ilustrar o significado deste teorema. Nas figuras $1.2,1.3$ e 1.4, foram simulados 10 grafos $E R_{n}(\lambda / n)$, com $p=\lambda / n$ fazendo crescer $n$, para três distintos valores de $\lambda$. Estes grafos foram obtidos com ajuda do pacote "igraph" no programa R-project. Na figura 1.2 é apresentado sequências de grafos com $\lambda$ constante. Note que o primeiro grafo é conectado, mas quando $n$ começa a crescer os grafos são desconectados. Então para $\lambda$ constante, podemos dizer que a sequência de grafos da figura 1.2 são desconectados com alta probabilidade. Então para ter grafos conectados com alta probabilidade precisamos que $\lambda$ cresça quando $n$ cresce.

Na figura 1.3, temos grafos aleatórios $\operatorname{com} \lambda=\log \sqrt{n}$, neste caso $\lambda$ cresce quando $n$ cresce. Mas vemos que todos os grafos são desconectados. Isto é devido a que $\lambda$ cresce muito mais devagar que $\log n$, ou seja, $\lambda-\log n \rightarrow-\infty$. Então, tendo em consideração o Teorema 1.2.9, podemos dizer que esta sequência de grafos é desconectado com alta probabilidade. Por outro lado na figura 1.4, temos grafos com $\lambda=2 \log n$, neste caso podemos observar que todos os grafos são conectados. Note que $\lambda$ depende de $n$ e cresce quando $n$ também cresce. Podemos observar também que $\lambda$ cresce mais rápido que $\log n$, isto é, $\lambda-\log n \rightarrow \infty$. Então seguindo Teorema 1.2.6, podemos dizer que esta sequência de grafos são conectados com alta probabilidade. 


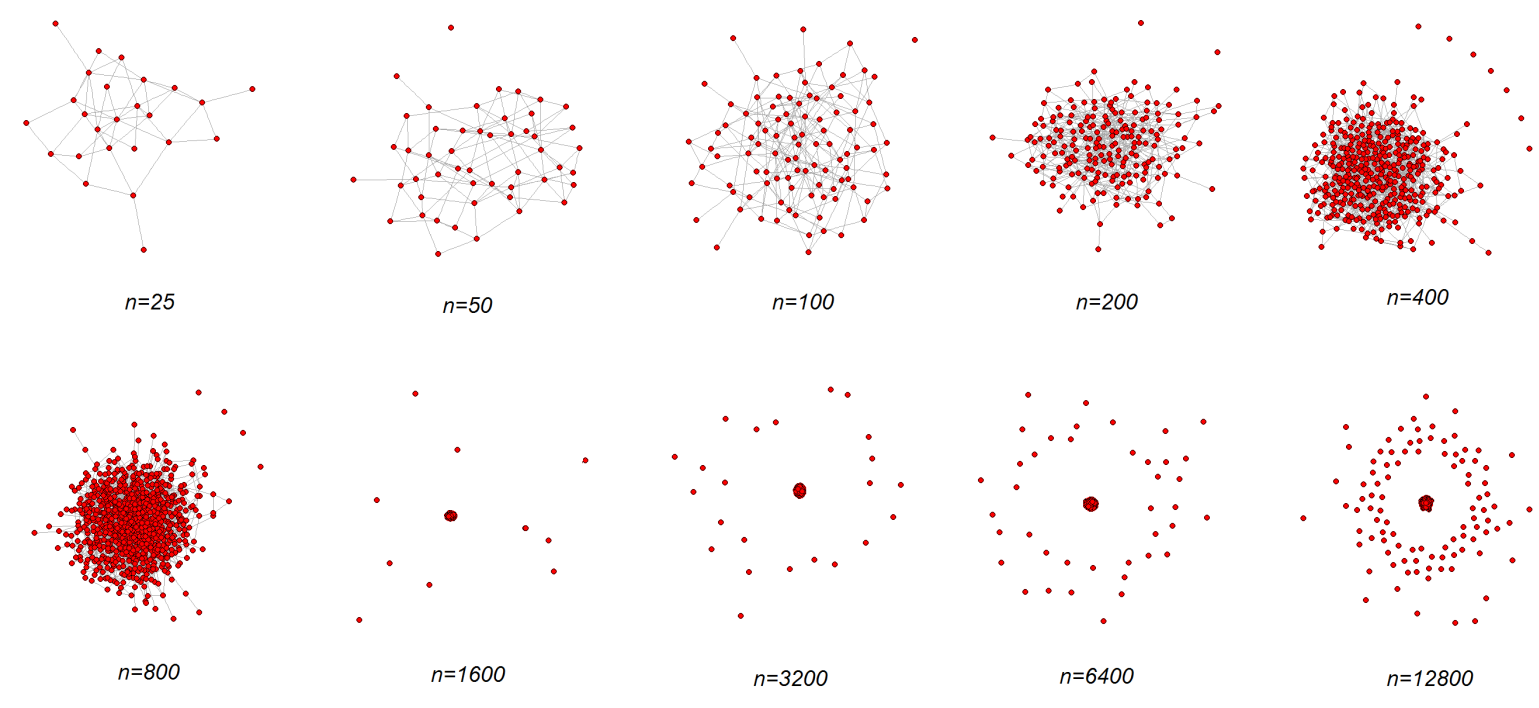

Figura 1.2: $\quad E R_{n}(\lambda / n)$ para $\lambda=5$ variando $n$.
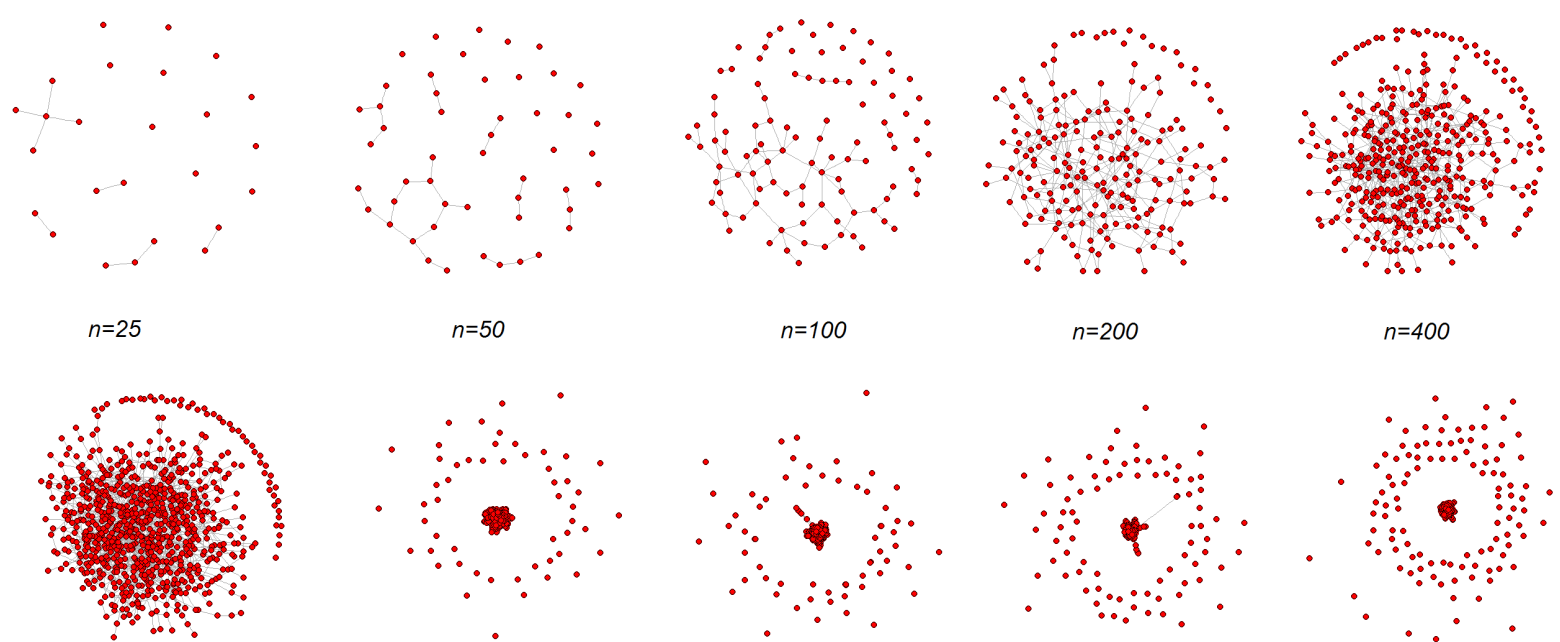

$n=800$
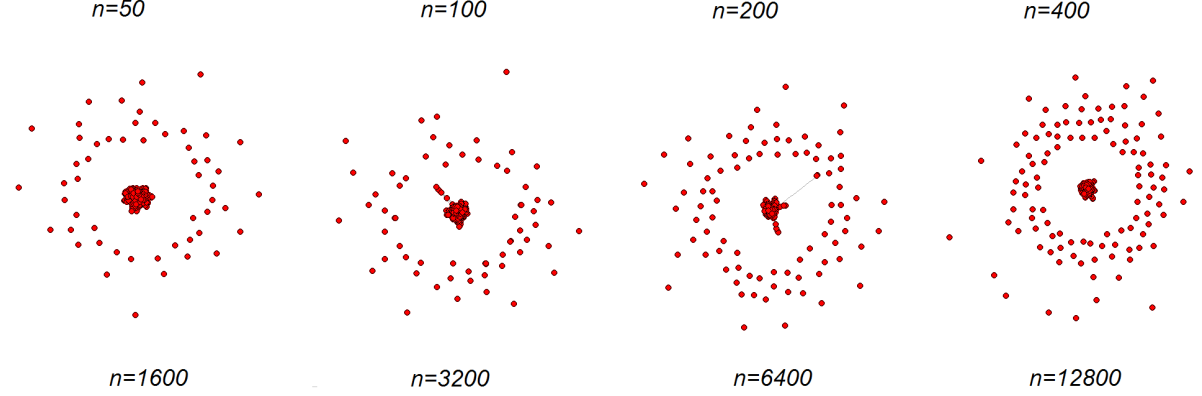

Figura 1.3: $\quad E R_{n}(\lambda / n)$ para $\lambda=\log \sqrt{n}$ variando $n$ 


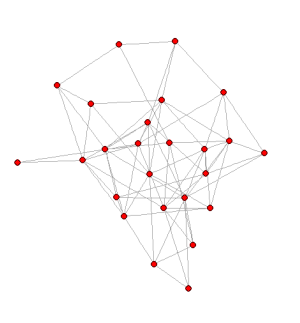

$n=25$

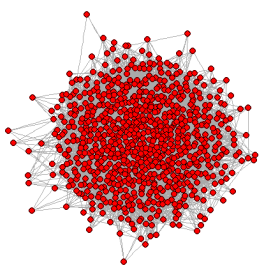

$n=800$

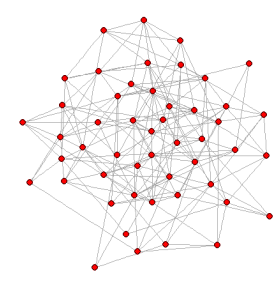

$n=50$

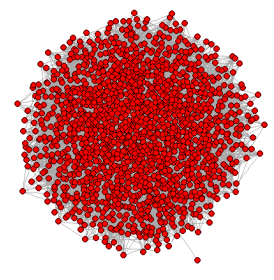

$n=1600$

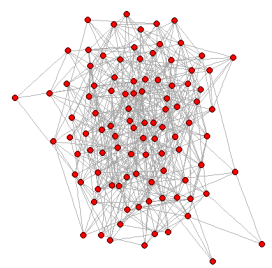

$n=100$

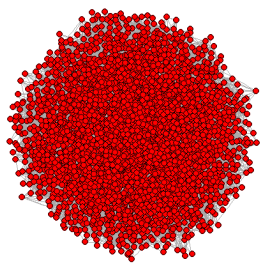

$n=3200$

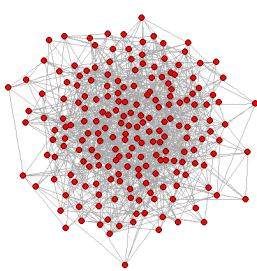

$n=200$

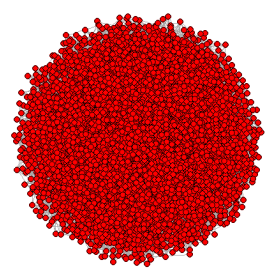

$n=6400$

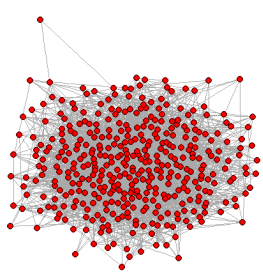

$n=400$

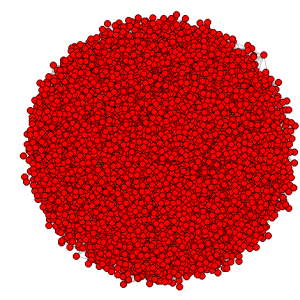

$n=12800$

Figura 1.4: $\quad E R_{n}(\lambda / n)$ para $\lambda=2 \log n$ variando $n$.

Na prova deste teorema vamos fazer uso do número de vértices isolados que vamos representar pela v.a $Y$, definida da seguinte forma

$$
Y=\sum_{i=1}^{n} I_{i}, \quad \text { onde } \quad I_{i}=\mathbb{1}_{\{|\mathcal{C}(i)|=1\}}
$$

em que $\mathcal{C}(i)$ representa a componente conectada do vértice $i$ e $|\mathcal{C}(i)|$ seu tamanho. Portanto $|\mathcal{C}(i)|=1$, quer dizer que o vértice $i$ não é conectado com nenhum outro vértice. Observe que $Y$ depende de $n$ mas por simplicidade não vamos incluir $n$ na notação. Claramente, quando $Y \geq 1$, existe pelo menos um vértice isolado, isto implica que o grafo é desconectado. Por outro lado, se $Y=0$ não existem vértices isolados, mas isto não implica que o grafo esteja conectado. No entanto, a Proposição 2.2.2 a seguir afirma que quando não há vértices isolados, o grafo aleatório é conectado com alta probabilidade. Para provar o Teorema 1.2.9 precisamos estudar a v.a $Y$, para o qual primeiro vamos estudar alguns métodos probabilísticos que são apresentados no capítulo seguinte. 


\subsection{Janela crítica para a conectividade}

No Teorema 1.2.9 é considerado a limiar da conectividade quando $\lambda-\log n \rightarrow-\infty$ e $\lambda-\log n \rightarrow \infty$. Mas o que acontece se $\lambda-\log n$ for constante? Ou seja, como seria o comportamento da conectividade de $E R_{n}(\lambda / n)$, se $\lambda-\log n=t, t \in \mathbb{R}$ fixo. Este fenômeno é chamado janela crítica para a conectividade e é explicado pelo Teorema 1.3.1 que apresentamos nesta seção. Para ver este teorema vamos considerar o grafo aleatório Erdös-Rényi de tamanho $n$ e probabilidade de conexão $p=\lambda / n$.

Teorema 1.3.1 (Fase crítica para a conectividade). Para $\lambda=\log n+t \rightarrow \infty$ o grafo aleatório Erdös-Rényi é conectado com probabilidade $e^{-e^{-t}}(1+o(1))$.

O ingrediente principal para provar este teorema é mostrar que, para $\lambda=\log n+t$, o número de vértices isolados $Y$ definido em (1.1), converge para uma variável aleatória Poisson com parâmetro $e^{-t}$. Esta prova é apresentada na seguinte seção.

Na Figura 1.5, foram simulados 10 grafos aleatórios Erdös-Rényi com $\lambda=\log n$, com o programa R-project. Neste caso veja que $\lambda-\log n=0$, de acordo com a figura apesar de que $n \rightarrow \infty$ nem sempre pode-se garantir a conectividade.

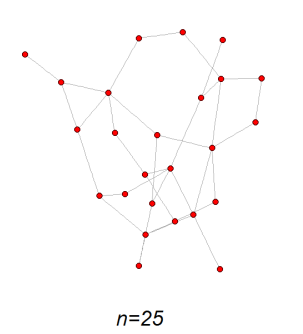

$n=25$

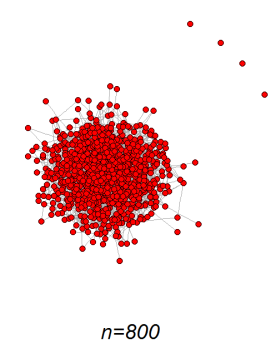

$n=800$
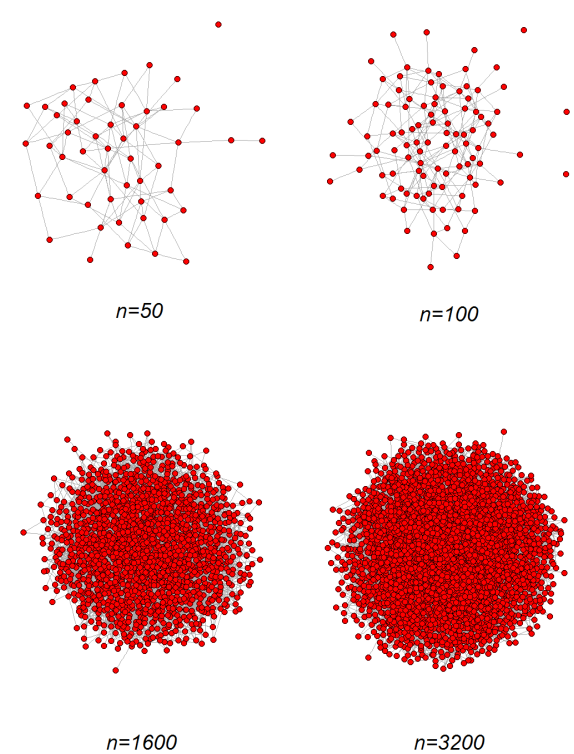

$n=3200$
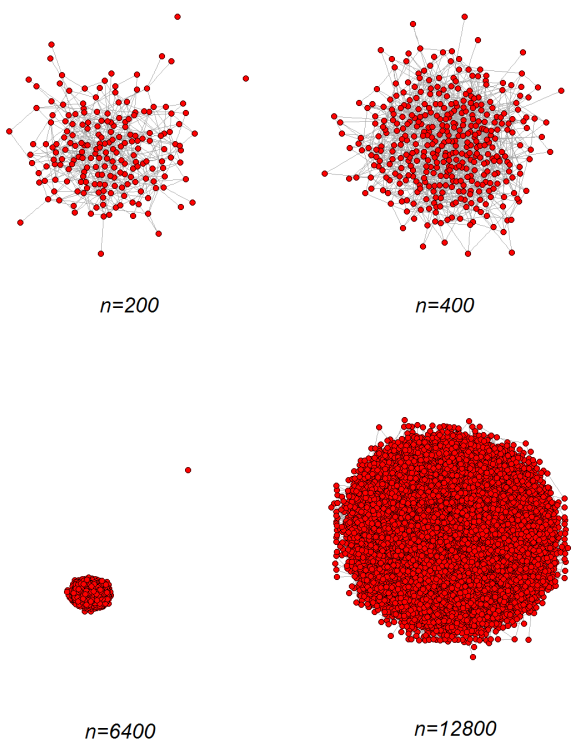

$n=12800$

Figura 1.5: $E R_{n}(\lambda / n)$ para $\lambda=\log n$ variando $n$. 


\section{Capítulo 2}

\section{Vértices isolados e conectividade de $E R_{n}(p)$}

\subsection{Métodos Probabilísticos}

O objetivo deste capítulo é revisar os métodos probabilísticos que serão úteis para o estudo de conectividade de grafos aleatórios considerados no presente trabalho. Primeiro estudamos a convergência para variáveis aleatórias Poisson que será uma ferramenta fundamental para mostrar a aproximação do número de vértices isolados para uma Poisson. Por fim na Seção 2.1.2 provamos duas desigualdades igualmente importantes para desenvolvimento deste trabalho.

\subsubsection{Convergência para uma variável aleatória Poisson}

No capítulo anterior vimos que no grafo aleatório $E R_{n}(p) \operatorname{com} p=\lambda / n$, para algum $\lambda>0$, a distribuição de grau é $\operatorname{Bin}(n-1, p)$. Quando $n$ é grande, e $n p=\lambda$ é fixada, então é bem conhecido que uma $\operatorname{Bin}(n-1, p)$ esta próximo de uma variável aleatória Poisson $(\lambda)$ com média $\lambda$. Para formalizar esta aproximação precisamos ter conhecimento de convergência de variáveis aleatórias, especialmente a convergência em distribuição. Antes de ver alguns critérios de convergencia em distribuiçõa veja a seguinte definição.

Definição 2.1.1 (Funções geradoras). Seja $X$ uma variável aleatória. Então

(a) A função característica de $X$ é definida por

$$
\phi_{X}(t)=E\left[e^{i t X}\right]
$$

desde que $\left|e^{i t X}\right|=1$ para todo $t$.

(b) A função geradora de probabilidade de uma v.a. $X$ de valores inteiros não negativos é definida por

$$
G_{X}(t)=E\left[t^{X}\right]
$$

pelo menos para $|t|<1$, desde que é uma série de potências com coeficientes em $[0,1]$. 
(c) A função geradora de momentos de $X$ é definida por

$$
M_{X}(t)=E\left[e^{t X}\right],
$$

desde que a esperança seja finita para $t \in \mathbb{R}$ em algum intervalo $-t_{0}<t<t_{0}$; com $t_{0}>0$.

A esperança pode nem sempre ser finita e, portanto, $M_{X}(t)$ nem sempre existirá. Para mostrar isto vejamos o seguinte exemplo.

Exemplo 2.1.2. Seja a v.a. $X$ com distribuição Cauchy e com função de densidade dada por

$$
f_{X}(x)=\frac{1}{\pi\left(1+x^{2}\right)}, \quad-\infty<x<\infty
$$

Para $t>0$, temos

$$
\begin{aligned}
M_{X}(t) & =\int_{-\infty}^{\infty} e^{t x} \frac{1}{\pi\left(1+x^{2}\right)} d x \\
& >\frac{1}{\pi} \int_{0}^{\infty} e^{t x} \frac{1}{\left(1+x^{2}\right)} d x \\
& >\frac{1}{\pi} \int_{0}^{\infty} t x \frac{1}{\left(1+x^{2}\right)} d x \text { pois } e^{x}>x \text { para } x>0 \\
& =\frac{t}{2 \pi} \int_{0}^{\infty} \frac{2 x}{\left(1+x^{2}\right)} d x \\
& =\frac{t}{2 \pi} \int_{1}^{\infty} \frac{1}{y} d y \text { fazendo } y=1+x^{2} \\
& =\infty
\end{aligned}
$$

Para $t<0$, usando os limites $-\infty$ e 0 também obtemos que $M_{X}(t)=\infty$. Então para $t \neq 0, M_{X}(t)=\infty$, portanto a função geradora de momentos para uma v.a. $X$ com distribuição Cauchy não existe.

Teorema 2.1.3 (Critério para convergência em distribuição). Seja $\mathbb{E}\left[X^{r}\right]$ o r-ésimo momento da v.a $X$. A sequência de variáveis aleatórias $\left\{X_{n}\right\}_{n=1}^{\infty}$ converge em distribuição a uma v.a $X$ :

(a) Se, e somente se, a função característica de $X_{n}, \phi_{X_{n}}(t)$ converge à função característica de $X, \phi_{X}(t)$, para todo $t$.

(b) Quando, para algum $\varepsilon>0$, a função geradora de momentos de $X_{n}, M_{X_{n}}(t)$ converge à função geradora de momentos de $X, M_{X}(t)$, para todo $|t|<\varepsilon$.

(c) Quando, para algum $\varepsilon>0$, a função geradora de probabilidade de $X_{n}, G_{X_{n}}(t)$ converge à função geradora de probabilidade de $X, G_{X}(t)$, para todo $|t|<1+\varepsilon$.

(d) Quando $X_{n}$ toma valores inteiros e não negativos, e $\mathbb{E}\left[X_{n}^{r}\right]$ convergem para $\mathbb{E}\left[X^{r}\right]$ para todo $r=1,2, \ldots$, desde que os momentos de $X, \mathbb{E}\left[X^{r}\right]$ satisfaçam

$$
\lim _{n \rightarrow \infty} \frac{\mathbb{E}\left[X^{r}\right] r^{m}}{r !}=0 \quad \forall m=0,1, \ldots
$$


(e) Quando $\mathbb{E}\left[X_{n}^{r}\right]$ converge para $\mathbb{E}\left[X^{r}\right]$, para todo $r=1,2$, . e a função geradora de momentos de $X, M_{X}(t)$ é finita para $t$ em alguma vizinhança da origem.

No seguinte exemplo mostraremos que uma variável aleatória Poisson satisfaz a condição de momentos em (2.1).

Exemplo 2.1.4. Seja $X \sim \operatorname{Poisson}(\lambda)$, a função geradora de momentos de $X$, é dada por

$$
M_{X}(t)=\mathbb{E}\left[e^{t X}\right]=e^{\lambda\left(e^{t}-1\right)}<\infty .
$$

Sabemos que

$$
M_{X}(t)=\sum_{x=0}^{\infty} e^{t x} \mathbb{P}[X=x],
$$

logo, usando a expansão de Taylor da exponencial, temos que

$$
M_{X}(t)=\sum_{x=0}^{\infty} \sum_{r=0}^{\infty} \frac{t^{r} x^{r}}{r !} \mathbb{P}[X=x],
$$

desde que $M_{X}(t)<\infty$ para todo $t$, temos que

$$
M_{X}(t)=\sum_{r=0}^{\infty} \mathbb{E}\left[X^{r}\right] \frac{t^{r}}{r !}<\infty,
$$

ou seja a série converge, então devemos ter que

$$
\lim _{r \rightarrow \infty} \mathbb{E}\left[X^{r}\right] \frac{t^{r}}{r !}=0
$$

Logo para $t=r>1$ temos

$$
\lim _{r \rightarrow \infty} \mathbb{E}\left[X^{r}\right] \frac{r^{r}}{r !}=0
$$

e para $r>m$ para todo $m=0,1,2, \ldots$ temos

$$
r^{r}>r^{m} \Rightarrow \mathbb{E}\left[X^{r}\right] \frac{r^{r}}{r !}>\mathbb{E}\left[X^{r}\right] \frac{r^{m}}{r !} \geq 0
$$

pois $\frac{\mathbb{E}\left[X^{r}\right]}{r !}>0$. Então

$$
\lim _{r \rightarrow \infty} \mathbb{E}\left[X^{r}\right] \frac{r^{r}}{r !}>\lim _{r \rightarrow \infty} \mathbb{E}\left[X^{r}\right] \frac{r^{m}}{r !} \geq 0,
$$

portanto por (2.2) concluímos que

$$
\lim _{r \rightarrow \infty} \mathbb{E}\left[X^{r}\right] \frac{r^{m}}{r !}=0
$$

Como a v.a $X$ com distribuição Poisson satisfaz a condição de momentos em (2.1), então para que uma sequência de v.a's $\left(x_{n}\right)_{n \geq 1}$ convergir em distribuição para $X$, basta mostrar que $\mathbb{E}\left[X_{n}^{r}\right]$ converge para $\mathbb{E}\left[X^{r}\right]$, para todo $r=1,2, \ldots$ 
Definição 2.1.5. Seja $r \in \mathbb{N}$, o $r$-ésimo momento factorial de uma v.a. $X$, que denotamos por $\mathbb{E}\left[(X)_{r}\right]$, é definida como:

$$
\mathbb{E}\left[(X)_{r}\right]=\mathbb{E}[X(X-1) \ldots(X-r+1)] .
$$

Exemplo 2.1.6. Seja $X$ uma variável aleatória Poisson com média $\lambda$, então

$$
\mathbb{E}\left[(X)_{r}\right]=\lambda^{r} .
$$

De fato,

$$
\begin{aligned}
\mathbb{E}\left[(X)_{r}\right] & =\mathbb{E}[X(X-1) \ldots(X-r+1)] \\
& =\sum_{x=0}^{\infty} x(x-1) \ldots(x-r+1) e^{-\lambda} \frac{\lambda^{x}}{x !}
\end{aligned}
$$

logo, note que para $x=0,1, \ldots, r-1$ temos que $\mathbb{E}\left[(X)_{r}\right]=0$, então

$$
\begin{aligned}
\mathbb{E}\left[(X)_{r}\right] & =\sum_{x=r}^{\infty} x(x-1) \ldots(x-r+1) e^{-\lambda} \frac{\lambda^{x}}{x !} \\
& =\lambda^{r} \sum_{x=r}^{\infty} \frac{x(x-1) \ldots(x-r+1) e^{-\lambda} \lambda^{x-r}}{x(x-1) \ldots(x-r+1)(x-r) !} \\
& =\lambda^{r} \sum_{x=r}^{\infty} \frac{e^{-\lambda} \lambda^{x-r}}{(x-r) !} \\
& =\lambda^{r} .
\end{aligned}
$$

Observação 2.1.7. Observe que o momento de uma variável aleatória $X \sim \operatorname{Poisson}(\lambda)$, satisfaz

$$
\mathbb{E}\left[X^{m}\right]=\lambda E\left[(X+1)^{m}-1\right] .
$$

De fato,

$$
\begin{aligned}
\mathbb{E}\left[X^{m}\right] & =\sum_{x=0}^{\infty} x^{m} \frac{\lambda^{x} e^{-\lambda}}{x !}=\lambda \sum_{x=1}^{\infty} x^{m} \frac{e^{-\lambda} \lambda^{x-1}}{x(x-1) !} \\
& =\lambda \sum_{x-1=0}^{\infty} x^{m-1} \frac{e^{-\lambda} \lambda^{x-1}}{(x-1) !} \text { se } x-1=k, \text { então } \\
& =\lambda \sum_{k=0}^{\infty}(k+1)^{m-1} \frac{e^{-\lambda} \lambda^{k}}{(k) !} \\
& =\lambda \mathbb{E}\left[(X+1)^{m-1}\right] .
\end{aligned}
$$

Por fim discutiremos um caso especial de convergência em distribuição, ou seja, quando tratamos com uma soma de indicadoras e o limite de uma variável aleatória Poisson. 
Para uma variável aleatória $X$ tomando valores em $\{0,1, \ldots, n\}$, o momento factorial de $X$ determina unicamente a função de probabilidade acumulada, desde que

$$
\mathbb{P}(X=k)=\sum_{r=k}^{n}(-1)^{r+k} \frac{\mathbb{E}\left[(X)_{r}\right]}{(r-k) ! k !} .
$$

Ver [2, cap. 1, pag. 18]. Para ver (2.4), escrevemos

$$
I_{\{X=k\}}=\left(\begin{array}{c}
X \\
k
\end{array}\right)(1-1)^{X-k}
$$

usando a convenção que $0^{0}=1$, e $\left(\begin{array}{l}n \\ k\end{array}\right)=0$, quando $k<0$ ou $k>n$, logo por binômio de Newton obtemos

$$
\begin{aligned}
I_{\{X=k\}} & =\left(\begin{array}{c}
X \\
k
\end{array}\right) \sum_{i=0}^{X-k}\left(\begin{array}{c}
X-k \\
i
\end{array}\right)(-1)^{i} \\
& =\sum_{i=0}^{X-k}\left(\begin{array}{c}
X \\
k
\end{array}\right)\left(\begin{array}{c}
X-k \\
i
\end{array}\right)(-1)^{i} \\
& =\sum_{i=0}^{n-k}\left(\begin{array}{c}
X \\
k
\end{array}\right)\left(\begin{array}{c}
X-k \\
i
\end{array}\right)(-1)^{i},
\end{aligned}
$$

assim

$$
\begin{aligned}
I_{\{X=k\}} & =\sum_{i=0}^{n-k}(-1)^{i} \frac{X !}{(X-k) ! k !} \frac{(X-k) !}{(X-k-i) ! i !} \\
& =\sum_{i=0}^{n-k}(-1)^{i} \frac{X(X-1) \ldots(X-k-i+1)(X-k-i) !}{(X-k-i) ! k ! i !} \\
& =\sum_{i=0}^{n-k}(-1)^{i} \frac{X(X-1) \ldots(X-k-i+1)}{k ! i !} \\
& =\sum_{i=0}^{n-k}(-1)^{i} \frac{(X)_{k+i}}{k ! i !} \\
& =\sum_{r=k}^{n}(-1)^{r+k} \frac{(X)_{r}}{k !(r-k) !} .
\end{aligned}
$$

Logo tomando valor esperado, temos

$$
\mathbb{P}(X=k)=\sum_{r=k}^{n}(-1)^{r+k} \frac{\mathbb{E}\left[(X)_{r}\right]}{k !(r-k) !} .
$$

Por outro lado, existe um resultado similar para variáveis aleatórias não limitadas, o qual obtem-se como segue. Supondo que $\mathbb{E}\left[(X)_{r}\right]$ é finito para $r=1, \ldots, R$, e fazendo 
$X^{(n)}=\min \{X, n\}$, temos que $\lim _{n \rightarrow \infty} \mathbb{E}\left[\left(X^{(n)}\right)_{r}\right]=\mathbb{E}\left[X_{r}\right]$ para $r=1, \ldots, R$ e para $k \leq n$ temos que $\mathbb{P}\left(X^{(n)}=k\right)=\mathbb{P}(X=k)$. Assim, da observação anterior e para $s, t \leq R, k+s$ ímpar e $k+t$ par, obtemos que

$$
\sum_{r=k}^{s}(-1)^{r+k} \frac{\mathbb{E}\left[(X)_{r}\right]}{k !(r-k) !} \leq \mathbb{P}(X=k) \leq \sum_{r=k}^{t}(-1)^{r+k} \frac{\mathbb{E}\left[(X)_{r}\right]}{k !(r-k) !} .
$$

Assim obtemos que

$$
\mathbb{P}(X=k)=\sum_{r=k}^{\infty}(-1)^{r+k} \frac{\mathbb{E}\left[(X)_{r}\right]}{k !(r-k) !} .
$$

Isto implica no seguinte resultado.

Teorema 2.1.8 (Convergência para uma variável aleatória Poisson). Uma sequência de variáveis aleatórias $\left\{X_{n}\right\}_{n=1}^{\infty}$ de valores inteiros, converge em distribuição para uma variável aleatória Poisson com parâmetro $\lambda$, se

$$
\lim _{n \rightarrow \infty} \mathbb{E}\left[\left(X_{n}\right)_{r}\right]=\lambda^{r} \quad \text { para todo } r=1,2, \ldots,
$$

O Teorema 2.1.8 é particularmente conveniente quando tratamos com soma de indicadores, ou seja, quando

$$
X_{n}=\sum_{i \in \mathcal{I}_{n}} I_{i, n}
$$

onde $I_{i, n}$ toma valores 0 e 1 , somente. Note que segundo (1.1) o número de vértices isolados pode ser escrito como soma de indicadoras.

Teorema 2.1.9 (Momentos fatoriais de soma de indicadoras). Quando $X=\sum_{i \in \mathcal{I}} I_{i}$ é uma soma de indicadoras, em que $\mathcal{I}$ é um conjunto finito de subindices, então

$$
\mathbb{E}\left[(X)_{r}\right]=\sum_{i_{1}, \ldots, i_{r} \in \mathcal{I}}^{*} \mathbb{E}\left[\prod_{l=1}^{r} I_{i_{l}}\right]=\sum_{i_{1}, \ldots, i_{r} \in \mathcal{I}}^{*} \mathbb{P}\left(I_{i_{1}}=\ldots=I_{i_{r}}=1\right)
$$

onde $\sum_{i_{1}, \ldots, i_{r} \in \mathcal{I}}^{*}$ denota uma soma em índices distintos.

Prova. Provaremos (2.7) por indução em $r \geq 1$ e para toda medida de probabilidade e suas correspondentes esperanças $\mathbb{E}$. Para $r=1$ temos que $(X)_{1}=X$ e (2.7) resulta do fato de que a esperança de uma soma de v.a. é a soma das esperanças das v.a. isto é, desde que

$$
X=\sum_{i \in \mathcal{I}} I_{i}
$$

então temos que

$$
\begin{aligned}
\mathbb{E}\left[(X)_{1}\right] & =\mathbb{E}[(X)]=\mathbb{E}\left[\sum_{i \in \mathcal{I}} I_{i}\right]=\sum_{i \in \mathcal{I}} \mathbb{E}\left[I_{i}\right] \\
& =\sum_{i \in \mathcal{I}}\left(\sum_{s=0}^{1} s \mathbb{P}\left[I_{i}=s\right]\right)=\sum_{i \in \mathcal{I}} \mathbb{P}\left[I_{i}=1\right]
\end{aligned}
$$


Isto inicia a hipótese indutiva. Continuando com a prova por indução, note que é suficiente provar a afirmação para indicadores $I_{i}$ que não são identicamente zero, isto é $\mathbb{P}\left[I_{i}=1\right]>0$. Então para $r \geq 2$ podemos escrever

$$
\begin{aligned}
\mathbb{E}\left[(X)_{r}\right] & =\mathbb{E}[X(X-1) \ldots(X-r+1)] \\
& =\mathbb{E}\left[\sum_{i_{1} \in \mathcal{I}} I_{i_{1}}(X-1) \ldots(X-r+1)\right] \\
& =\sum_{i_{1} \in \mathcal{I}} \mathbb{E}\left[I_{i_{1}}(X-1) \ldots(X-r+1)\right] .
\end{aligned}
$$

Denotando por $\mathbb{P}_{i_{1}}(A)$ a distribuição condicional "dado que $I_{i_{1}}=1$ ", isto é, para quaisquer evento $A$, tem-se que

$$
\mathbb{P}_{i_{1}}(A)=\frac{\mathbb{P}\left(A \cap\left\{I_{i_{1}}=1\right\}\right)}{\mathbb{P}\left(I_{i_{1}}=1\right)}
$$

ou equivalentemente

$$
\mathbb{P}_{i_{1}}(A) \mathbb{P}\left(I_{i_{1}}=1\right)=\mathbb{P}\left(A \cap\left\{I_{i_{1}}=1\right\}\right) .
$$

Então, fazendo $Z=(X-1) \ldots(X-r+1)$, podemos escrever

$$
\begin{aligned}
\mathbb{E}\left[I_{i_{1}}(X-1) \ldots(X-r+1)\right] & =\sum_{s, z} s z \mathbb{P}\left(I_{i_{1}}=s, Z=z\right) \quad ; s=0,1 \\
& =\sum_{z}\left(1 z \mathbb{P}\left(I_{i_{1}}=1, Z=z\right)+0 z \mathbb{P}\left(I_{i_{1}}=0, Z_{z}\right)\right) \\
& =\sum_{z} z \mathbb{P}\left(I_{i_{1}}=1, Z=z\right)=\sum_{z} z \mathbb{P}\left(Z=z / I_{i_{1}}=1\right) \mathbb{P}\left(I_{i_{1}}=1\right) \\
& =\mathbb{P}\left(I_{i_{1}}=1\right) \sum_{z} z \mathbb{P}_{i_{1}}(Z=z) \\
& =\mathbb{P}\left(I_{i_{1}}=1\right) \mathbb{E}_{i_{1}}[Z] \\
& =\mathbb{P}\left(I_{i_{1}}=1\right) \mathbb{E}[(X-1) \ldots(X-r+1)] .
\end{aligned}
$$

Defina

$$
Y=X-I_{i_{1}}=\sum_{j \in \mathcal{I} \backslash\left\{i_{1}\right\}} I_{j}
$$

e note que condicionalmente em $I_{i_{1}}=1$, temos que $X=Y+1$ e como resultado obtemos que

$$
\mathbb{E}_{i_{1}}[(X-1) \ldots(X-r+1)]=\mathbb{E}_{i_{1}}[Y \ldots(Y-r+2)]=\mathbb{E}_{i_{1}}\left[(Y)_{r-1}\right] .
$$

Aplicando a hipótese indutiva para $\mathbb{E}\left[(Y)_{r-1}\right]$, obtemos que

$$
\mathbb{E}_{i_{1}}\left[(Y)_{r-1}\right]=\sum_{i_{2}, \ldots, i_{r} \in \mathcal{I} \backslash\left\{i_{1}\right\}}^{*} \mathbb{P}_{i_{1}}\left(I_{i_{2}}=\ldots=I_{i_{r}}=1\right)
$$

Como resultado, chegamos a

$$
\mathbb{E}\left[(X)_{r}\right]=\sum_{i_{1} \in \mathcal{I}} \mathbb{P}\left(I_{i_{1}}=1\right) \sum_{i_{2}, \ldots, i_{r} \in \mathcal{I} \backslash\left\{i_{1}\right\}}^{*} \mathbb{P}_{i_{1}}\left(I_{i_{2}}=\ldots=I_{i_{r}}=1\right)
$$


Agora completamos a prova notando que

$$
\mathbb{P}\left(I_{i_{1}}=1\right) . \mathbb{P}_{i_{1}}\left(I_{i_{2}}=\ldots=I_{i_{r}}=1\right)=\mathbb{P}\left(I_{i_{1}}=I_{i_{2}}=\ldots=I_{i_{r}}=1\right)
$$

e que

$$
\sum_{i_{1} \in \mathcal{I}} \sum_{i_{2}, \ldots, i_{r} \in \mathcal{I} \backslash\left\{i_{1}\right\}}^{*}=\sum_{i_{1}, \ldots, i_{r} \in \mathcal{I}}^{*}
$$

Assim

$$
\mathbb{E}\left[(X)_{r}\right]=\sum_{i_{1}, \ldots, i_{r} \in \mathcal{I}} \mathbb{P}\left(I_{i_{1}}=\ldots=I_{i_{r}}=1\right)
$$

Observação 2.1.10. Considere uma sequência de v.a's binomiais $\left\{X_{n}\right\}_{n \in \mathbb{N}}$ com parâmetros $n$ e $p=\lambda / n$ que pode ser escrito como uma soma de variáveis aleatórias $I_{i}$ Bernoulli independentes, isto é

$$
X_{n}=\sum_{i=1}^{n} I_{i}
$$

pelo Teorema 2.1.9 temos

$$
\mathbb{E}\left[\left(X_{n}\right)_{r}\right]=\sum_{i_{1}, \ldots, i_{r} \in \mathcal{I}}^{*} \mathbb{P}\left(I_{i_{1}}=\ldots=I_{i_{r}}=1\right),
$$

em que $\mathcal{I}=\{1, \ldots, n\}$. Logo pela independência das v.a's $I_{i}$ e desde que $\sum_{i_{1}, \ldots, i_{r} \in \mathcal{I}}^{*}$ denota uma soma de índices distintos podemos escrever

$$
\mathbb{E}\left[\left(X_{n}\right)_{r}\right]=n(n-1) \ldots(n-r+1) \prod_{j=1}^{r} \mathbb{P}\left(I_{i_{j}}=1\right)=n(n-1) \ldots(n-r+1) p^{r} .
$$

Aplicando limite e fazendo $p=\lambda / n$ obtemos

$$
\lim _{n \rightarrow \infty} \mathbb{E}\left[\left(X_{n}\right)_{r}\right]=\lim _{n \rightarrow \infty} n(n-1) \ldots(n-r+1)\left(\frac{\lambda}{n}\right)^{r}=\lambda^{r} .
$$

Pelo Teorema 2.1 .8 concluímos que uma sequência de v.a's $\left\{X_{n}\right\}_{n \in \mathbb{N}}$ com distribuição Binomial de parâmetros $n$ e $p=\lambda / n$ converge em distribuição a uma v.a Poisson $X$ com parâmetro $\lambda$.

\subsubsection{Métodos do primeiro e segundo momento}

Nesta seção apresentaremos duas desigualdades que são ferramentas muito importantes na teoria de probabilidades e que serão úteis para o desenvolvimento deste trabalho.

Proposição 2.1.11 (Desigualdade de Markov). Se X é uma v. a não negativa com $\mathbb{E}[X]<$ $\infty$, então para qualquer $a>0$ tem-se que

$$
\mathbb{P}(X \geq a) \leq \frac{\mathbb{E}[X]}{a} .
$$

Em particular, quando $X$ possui valores inteiros e $m>0$ é tal que $\mathbb{E}[X] \leq m$, então

$$
\mathbb{P}(X=0) \geq 1-m .
$$


Prova. Para provar (2.8) note que $a \mathbb{1}_{\{X \geq a\}} \leq X$, então

$$
\mathbb{E}\left[a \mathbb{1}_{\{X \geq a\}}\right] \leq \mathbb{E}[X]
$$

Agora, o lado esquerdo desta desigualdade coincide com

$$
a \mathbb{E}\left[\mathbb{1}_{\{X \geq a\}}\right]=a \mathbb{P}(X \geq a),
$$

e portanto

$$
\mathbb{P}(X \geq a) \leq \frac{\mathbb{E}[X]}{a} .
$$

Para provar (2.9) considere $a=1$, logo por (2.8) e desde que $\mathbb{E}[X] \leq m$, então

$$
\mathbb{P}(X \geq 1) \leq \mathbb{E}[X] \leq m
$$

Logo

$$
1-\mathbb{P}(X \geq 1) \geq 1-m
$$

que é equivalente a escrever

$$
\mathbb{P}(X=0) \geq 1-m,
$$

desde que $X$ toma valores inteiros não negativos.

Proposição 2.1.12 (Desigualdade de Chebychev). Seja $X$ uma v.a. de valores inteiros com $\operatorname{Var}(X)=\sigma^{2}$, então para qualquer $a>0$ tem-se que

$$
\mathbb{P}(|X-\mathbb{E}[X]| \geq a) \leq \frac{\sigma^{2}}{a^{2}}
$$

Em particular, quando $X$ toma valores inteiros com $\mathbb{E}[X] \geq m, m>0$ e $\operatorname{Var}(X)=\sigma^{2}$, então

$$
\mathbb{P}(X=0) \leq \frac{\sigma^{2}}{m^{2}}
$$

Prova. Para (2.10), note que

$$
\mathbb{P}(|X-\mathbb{E}[X]| \geq a)=\mathbb{P}\left((X-\mathbb{E}[X])^{2} \geq a^{2}\right)
$$

e aplicando a Desigualdade de Markov obtemos

$$
\mathbb{P}(|X-\mathbb{E}[X]| \geq a) \leq \frac{\operatorname{Var}(X)}{a^{2}} .
$$

Para provar (2.11), note que

$$
\mathbb{P}(X=0) \leq \mathbb{P}(|X-\mathbb{E}[X]| \geq \mathbb{E}[X]) \leq \frac{\operatorname{Var}(X)}{\mathbb{E}[X]^{2}}
$$

e desde que $\mathbb{E}[X] \geq m$, então

$$
\mathbb{P}(X=0) \leq \frac{\sigma^{2}}{m^{2}}
$$


Podemos encontrar estas provas em [4, cap. 8, pag. 459-560].

Observação 2.1.13.

- Por (2.9), se a v.a inteira $X$ tem uma média pequena, então $X=0$ com maior probabilidade. Isto é chamado o Método do Primeiro Momento, e é uma ferramenta poderosa para provar resultados.

- Por (2.11), se a v.a inteira $X$ tem uma média grande, e uma variância que é pequena em comparação com o quadrado da média, então $X>0$ com maior probabilidade. Isto é chamado o Método do Segundo Momento.

\subsection{Prova do Teorema de limiar da Conectividade para $E R_{n}(p)$}

Como foi explicado na Seção 1.2, para provar o Teorema 1.2.9, vamos estudar a v.a $Y$ que representa o número de vértices isolados,

$$
Y:=\sum_{i=1}^{n} I_{i}, \quad \text { onde } \quad I_{i}:=\mathbb{1}_{\{|\mathcal{C}(i)|=1\}},
$$

em que $\mathcal{C}(i)$ representa a componente conectada incluindo o vértice $i$ e $|\mathcal{C}(i)|$ seu tamanho. A prova é baseada nas Proposições 2.2.1 e 2.2.2.

\subsubsection{Controle dos momentos do número de vértices isolados}

No caso onde $|\lambda-\log n| \rightarrow \infty$, usamos as Proposições 2.1.11 e 2.1 .12 assim como o primeiro e segundo momento usando a variância de $Y$ na seguinte proposição.

Proposição 2.2.1 (média e variância do número de vértices isolados). Para todo $\lambda \leq n / 2$ $\operatorname{com} \lambda \geq 1 / 2$,

$$
\mathbb{E}_{\lambda}[Y]=n e^{-\lambda}\left(1+O\left(\lambda^{2} / n\right)\right)
$$

e para todo $\lambda \leq n$,

$$
\operatorname{Var}_{\lambda}(Y) \leq \mathbb{E}_{\lambda}[Y]+\frac{\lambda}{n-\lambda} \mathbb{E}_{\lambda}[Y]^{2} .
$$

Prova. Veja que, por (1.1) temos que

$$
\mathbb{E}_{\lambda}[Y]=\mathbb{E}_{\lambda}\left[\sum_{i=1}^{n} I_{i}\right]=n \mathbb{P}_{\lambda}(|\mathcal{C}(i)|=1)=n\left(1-\frac{\lambda}{n}\right)^{n-1}
$$

Usando $1-x \leq e^{-x}$ e a série de Taylor da exponencial, temos 


$$
\begin{aligned}
\mathbb{E}_{\lambda}[Y] & \leq n\left(e^{-\lambda / n}\right)^{n-1} \\
& =n e^{-\lambda} e^{\lambda / n} \\
& =n e^{-\lambda}\left(\sum_{k=0}^{\infty} \frac{(\lambda / n)^{k}}{k !}\right) \\
& =n e^{-\lambda}\left(1+\frac{\lambda}{n} \sum_{k=1}^{\infty} \frac{(\lambda / n)^{k-1}}{k !}\right) .
\end{aligned}
$$

Desde que

$$
\sum_{k=1}^{\infty} \frac{(\lambda / n)^{k-1}}{k !} \leq \sum_{k=1}^{\infty} \frac{(\lambda)^{k-1}}{k !}<\infty
$$

para $n$ suficientemente grande (ou de fato para todo $n \geq 2$ ), temos que

$$
\mathbb{E}_{\lambda}[Y] \leq n e^{-\lambda}(1+O(\lambda / n)) .
$$

Da mesma forma, usando $1-x \geq e^{-x-x^{2}}$ para $0 \leq x \leq 1 / 2$, obtemos

$$
\begin{aligned}
\mathbb{E}_{\lambda}[Y] & \geq n\left(e^{-\frac{\lambda}{n}-\left(\frac{\lambda}{n}\right)^{2}}\right)^{n-1} \\
& =n e^{-(n-1) \frac{\lambda}{n}\left(1+\frac{\lambda}{n}\right)} \\
& \geq n e^{-\lambda} e^{-\lambda^{2} / n} \\
& \geq n e^{-\lambda}\left(1+\left(-\frac{\lambda^{2}}{n}\right)\right) .
\end{aligned}
$$

Assim

$$
\mathbb{E}_{\lambda}[Y] \geq n e^{-\lambda}\left(1+O\left(\lambda^{2} / n\right)\right) .
$$

E desde que $\lambda \geq 1 / 2$, temos que $O(\lambda / n)=O\left(\lambda^{2} / n\right)$, então a média do número de vértices isolados é

$$
\mathbb{E}_{\lambda}[Y]=n e^{-\lambda}\left(1+O\left(\lambda^{2} / n\right)\right) .
$$

Por outro lado, por convenção para $\lambda=n$, $\operatorname{Var}_{\lambda}(Y)=0$, logo o lado direito da desigualdade (2.13) é igual a infinito. Então para $\lambda=n,(2.13)$ é verdade. Basta provar esta desigualdade para $\lambda<n$. Da permutabilidade dos vértices e pelo Teorema 2.1.9 temos que

$$
\mathbb{E}_{\lambda}\left[(Y)_{2}\right]=\frac{n !}{(n-2)} \mathbb{P}_{\lambda}\left(I_{1}=I_{2}=1\right)=n(n-1) \mathbb{P}_{\lambda}(|\mathcal{C}(1)|=1,|\mathcal{C}(2)|=1) .
$$

Lembrando que $(Y)_{r}=Y(Y-1) \ldots(Y-r+1)$ e $\mathbb{E}_{\lambda}\left[(Y)_{r}\right]$ representa o $r$-ésimo momento factorial de $Y$, então para $r=2$ temos que

$$
\mathbb{E}_{\lambda}\left[(Y)_{2}\right]=\mathbb{E}_{\lambda}\left[Y^{2}\right]-\mathbb{E}_{\lambda}[Y]
$$

Assim

$$
\mathbb{E}_{\lambda}\left[Y^{2}\right]=n \mathbb{P}_{\lambda}(|\mathcal{C}(1)|=1)+n(n-1) \mathbb{P}_{\lambda}(|\mathcal{C}(1)|=1,|\mathcal{C}(2)|=1)
$$


em que

$$
\begin{aligned}
\mathbb{P}_{\lambda}(|\mathcal{C}(1)|=1,|\mathcal{C}(2)|=1) & =\mathbb{P}_{\lambda}(|\mathcal{C}(1)|=1) \mathbb{P}_{\lambda}(|\mathcal{C}(2)|=1 /|\mathcal{C}(1)|=1) \\
& =\left(1-\frac{\lambda}{n}\right)^{n-1}\left(1-\frac{\lambda}{n}\right)^{n-2} \\
& =\left(1-\frac{\lambda}{n}\right)^{2 n-3}
\end{aligned}
$$

Logo sabemos que $\operatorname{Var}_{\lambda}(Y)=\mathbb{E}_{\lambda}\left[Y^{2}\right]-\mathbb{E}_{\lambda}[Y]^{2}$, então

$$
\begin{aligned}
\operatorname{Var}_{\lambda}(Y) & =n \mathbb{P}_{\lambda}(|\mathcal{C}(1)|=1)+n(n-1) \mathbb{P}_{\lambda}(|\mathcal{C}(1)|=1,|\mathcal{C}(2)|=1)-n^{2} \mathbb{P}_{\lambda}(|\mathcal{C}(1)|=1)^{2} \\
& =n \mathbb{P}_{\lambda}(|\mathcal{C}(1)|=1)-n \mathbb{P}_{\lambda}(|\mathcal{C}(1)|=1,|\mathcal{C}(2)|=1) \\
& +n^{2}\left[\mathbb{P}_{\lambda}(|\mathcal{C}(1)|=1,|\mathcal{C}(2)|=1)-\mathbb{P}_{\lambda}(|\mathcal{C}(1)|=1)^{2}\right]
\end{aligned}
$$

Logo por (2.16) e usando o resultado $\mathbb{P}_{\lambda}(|\mathcal{C}(1)|=1)=\left(1-\frac{\lambda}{n}\right)^{n-1}$ obtemos

$$
\mathbb{P}_{\lambda}(|\mathcal{C}(1)|=1,|\mathcal{C}(2)|=1)-\mathbb{P}_{\lambda}(|\mathcal{C}(1)|=1)^{2}=\frac{\lambda}{n-\lambda} \mathbb{P}_{\lambda}(|\mathcal{C}(1)|=1)^{2}
$$

Assim

$$
\begin{aligned}
\operatorname{Var}_{\lambda}(Y) & =n \mathbb{P}_{\lambda}(|\mathcal{C}(1)|=1)-n \mathbb{P}_{\lambda}(|\mathcal{C}(1)|=1,|\mathcal{C}(2)|=1)+\frac{\lambda}{n-\lambda} n^{2} \mathbb{P}_{\lambda}(|\mathcal{C}(1)|=1)^{2} \\
& =\mathbb{E}_{\lambda}[Y]+\frac{\lambda}{n-\lambda} \mathbb{E}_{\lambda}[Y]^{2}-n \mathbb{P}_{\lambda}(|\mathcal{C}(1)|=1,|\mathcal{C}(2)|=1) .
\end{aligned}
$$

Portanto como $n \mathbb{P}_{\lambda}(|\mathcal{C}(1)|=1,|\mathcal{C}(2)|=1) \geq 0$ concluímos que

$$
\operatorname{Var}_{\lambda}(Y) \leq \mathbb{E}_{\lambda}[Y]+\frac{\lambda}{n-\lambda} \mathbb{E}_{\lambda}[Y]^{2}
$$

Isto prova a Proposição 2.2.1.

\subsubsection{Controlar $\mathbb{P}_{\lambda}(Y=0)$ basta para estudar a conectividade}

Proposição 2.2.2 (Conectividade e vértices isolados). Para todo $1 \leq \lambda \leq n$ e $n \geq 2$,

$$
\mathbb{P}_{\lambda}\left(E R_{n}(\lambda / n) \text { conectado }\right) \leq \mathbb{P}_{\lambda}(Y=0) .
$$

Por outro lado, se existe um $a>1 / 2$ tal que $\lambda \geq a \log n$, então, para $n \rightarrow \infty$,

$$
\mathbb{P}_{\lambda}\left(E R_{n}(\lambda / n) \text { conectado }\right)=\mathbb{P}_{\lambda}(Y=0)+o(1) .
$$

Prova. Note que o evento $\{Y>0\}$ que é dado por $\{\{|\mathcal{C}(1)|=1\} \cup\{|\mathcal{C}(2)|=1\} \cup \ldots \cup$ $\{|\mathcal{C}(n)|=1\}\}$, está contido no evento $\left\{E R_{n}(\lambda / n)\right.$ desconectado $\}$, assim

$$
\mathbb{P}_{\lambda}(Y>0) \leq \mathbb{P}_{\lambda}\left(E R_{n}(\lambda / n) \text { desconectado }\right)
$$


que é equivalente a

$$
1-\mathbb{P}_{\lambda}(Y=0) \leq 1-\mathbb{P}_{\lambda}\left(E R_{n}(\lambda / n) \text { conectado }\right)
$$

portanto

$$
\mathbb{P}_{\lambda}\left(E R_{n}(\lambda / n) \text { conectado }\right) \leq \mathbb{P}_{\lambda}(Y=0)
$$

Por outro lado, para provar $(2.18)$, veja que $\mathbb{P}_{\lambda}(Y \geq 0)=1$, então

$$
\begin{aligned}
\mathbb{P}_{\lambda}\left(E R_{n}(\lambda / n) \text { desconectado }\right) & =\mathbb{P}_{\lambda}\left(\left\{E R_{n}(\lambda / n) \text { desconectado }\right) \cap\{Y \geq 0\}\right\} \\
& =\mathbb{P}_{\lambda}\left(\left\{E R_{n}(\lambda / n) \text { desconectado }\right\} \cap[\{Y>0\} \cup\{Y=0\}]\right) \\
& =\mathbb{P}_{\lambda}\left(\left\{E R_{n}(\lambda / n) \text { desconectado }\right\} \cap\{Y>0\}\right) \\
& +\mathbb{P}_{\lambda}\left(\left\{E R_{n}(\lambda / n) \text { desconectado }\right\} \cap\{Y=0\}\right) .
\end{aligned}
$$

Como $\left\{E R_{n}(\lambda / n)\right.$ desconectado $\} \supset\{Y>0\}$, então

$\mathbb{P}_{\lambda}\left(E R_{n}(\lambda / n)\right.$ desconectado $)=\mathbb{P}_{\lambda}(Y>0)+\mathbb{P}_{\lambda}\left(\left\{E R_{n}(\lambda / n)\right.\right.$ desconectado $\left.\} \cap\{Y=0\}\right)$.

Usando este resultado, veja que basta provar que

$$
\mathbb{P}_{\lambda}\left(\left\{E R_{n}(\lambda / n) \text { desconectado }\right\} \cap\{Y=0\}\right)
$$

é uma função $o(1)$, para isto vamos considerar cálculos que envolvem árvores, para entender melhor esta parte veja o Apêndice A4, ou pode consultar [10, 11].

Veja que uma componente conectada pode ser representada como uma árvore, ou mais de uma dependendo se a componente contem ciclos ou não. Então considere um grafo que pode conter mais de uma componente conectada, mas não contém vértices isolados. Note que cada uma dessas componentes podem ser representadas por uma árvore, então no grafo podemos encontrar mais de uma árvore do mesmo tamanho.

Considere $X_{k}$ como sendo o número de árvores ocupadas de tamanho $k$ nos vértices $1, \ldots, n$, que não pode ser estendido para árvores de maior tamanho, para $k=2, \ldots, n$. Assim, cada árvore que é contado em $X_{k}$ tem tamanho igual a $k$, se denotamos seus vértices por $v_{1}, \ldots, v_{k}$, então todas as arestas entre $v_{i}, \forall i \in\{1, \ldots, k\}$ e $v \notin\left\{v_{1}, \ldots, v_{k}\right\}$ estão vazias. além disso, existem $k-1$ arestas ocupadas entre os $v_{i}$ vértices, com $i \in\{1, \ldots, k\}$, de tal forma que eles junto com as $k-1$ arestas formam uma árvore. Note que uma componente conectada de tamanho $k$, por conter mais de uma árvore de tamanho $k$, desde que a componente conectada pode conter ciclos. Além disso, quando $E R_{n}(\lambda / n)$ é desconectado e $Y=0$, deve haver um $k \in\{2, \ldots, n / 2\}$ para o qual $X_{k} \geq 1$. Com tudo isto podemos afirmar que

$$
\mathbb{P}_{\lambda}\left(E R_{n}(\lambda / n) \text { desconectado, } Y=0\right) \leq \mathbb{P}_{\lambda}\left(\bigcup_{k=2}^{n / 2}\left\{X_{k} \geq 1\right\}\right)
$$


Da desigualdade de Boole (ver Apêndice A1.1.) e da desigualdade de Markov, na Proposição 2.1.11, obtemos que

$$
\mathbb{P}_{\lambda}\left(E R_{n}(\lambda / n) \text { desconectado, } Y=0\right) \leq \sum_{k=2}^{n / 2} \mathbb{P}_{\lambda}\left(\left\{X_{k} \geq 1\right\}\right) \leq \sum_{k=2}^{n / 2} \mathbb{E}_{\lambda}\left[X_{k}\right]
$$

Para encontrar o valor de $\mathbb{E}_{\lambda}\left[X_{k}\right]$, observe que existem $\left(\begin{array}{l}n \\ k\end{array}\right)$ formas de escolher $k$ vértices e pelo Teorema de Cayley, no Apêndice A4.2., temos que o número de árvores marcados contendo $\mathrm{k}$ vértices, é $k^{k-2}$, com isto

$$
\mathbb{E}_{\lambda}\left[X_{k}\right]=\left(\begin{array}{l}
n \\
k
\end{array}\right) k^{k-2} q_{k}
$$

A notação $q_{k}$ representa a probabilidade de que alguma árvore de tamanho $k$ está ocupada e todas as arestas da árvore para os outros vértices, estão vazias, e é dado por

$$
q_{k}=\left(\frac{\lambda}{n}\right)^{k-1}\left(1-\frac{\lambda}{n}\right)^{k(n-k)}
$$

Do resultado (2.19), veja que, basta limitar $\mathbb{E}_{\lambda}\left[X_{k}\right]$, para isto, usamos $1-x \leq e^{-x}$, isto é

$$
\begin{aligned}
\mathbb{E}_{\lambda}\left[X_{k}\right] & \leq \frac{n !}{(n-k) ! k !} k^{k-2}\left(\frac{\lambda}{n}\right)^{k-1} e^{-\lambda k(n-k) / n} \\
& =\frac{n(n-1) \ldots(n-k+1)}{n^{k}} n \lambda^{k-1} \frac{k^{k-2}}{k !} e^{-\lambda k(n-k) / n} \\
& \leq n \lambda^{k-1} \frac{k^{k-2}}{k !} e^{-\lambda k(n-k) / n},
\end{aligned}
$$

pois $n(n-1) \ldots(n-k+1) \leq n^{k}$. Se além disso, usamos que $k ! \geq k^{k} e^{-k}$, pelo Limite de Stirling, e $\lambda \geq 1$, então obtemos

$$
\mathbb{E}_{\lambda}\left[X_{k}\right] \leq n(e \lambda)^{k} \frac{1}{k^{2}} e^{-\lambda k(n-k) / n} \leq n(e \lambda)^{k} e^{-\lambda k(n-k) / n} .
$$

Desde que $\lambda \longmapsto e^{-\lambda k(n-k) / n}$ é decrescente em $\lambda$, então para $\lambda=a \log n$, sendo algum $a>1 / 2$, tem-se que

$$
e^{-\lambda k(n-k) / n} \leq e^{-(a \log n) k(n-k) / n}=e^{-(a \log n) k} e^{\left(a k^{2} \log n\right) / n} .
$$

Logo veja que $\frac{a k^{2} \log n}{n}=o(1)$, pois $\lim _{n \rightarrow \infty} \frac{a k^{2} \log n}{n}=0$, ver Apêndice A3. Desta forma para $k \in\{2,3,4\}$, para $\lambda=a \log n$, para algum $a>1 / 2$, temos

$$
\mathbb{E}_{\lambda}\left[X_{k}\right] \leq n(e \lambda)^{4} e^{-\lambda k} e^{o(1)}=o(1)
$$

Por outro lado para $5 \leq k \leq n / 2$ e usando que $k(n-k) \geq k n / 2$, obtemos que

$$
\mathbb{E}_{\lambda}\left[X_{k}\right] \leq n\left(e \lambda e^{-\lambda / 2}\right)^{k}
$$


Como um resultado, para $\lambda=a \log n \operatorname{com} a>1 / 2$, e para todo $k \geq 5$, e usando que $\lambda \longmapsto \lambda e^{-\lambda / 2}$ é decrescente para $\lambda \geq 2$, temos

$$
\mathbb{E}_{\lambda}\left[X_{k}\right] \leq n^{1-k / 4}
$$

então por (2.19), (2.20) e 2.21 temos

$$
\mathbb{P}_{\lambda}\left(E R_{n}(\lambda / n) \text { desconectado, } Y=0\right) \leq o(1)+\sum_{k=5}^{n / 2} \mathbb{E}_{\lambda}\left[X_{k}\right] \leq o(1)+\sum_{k=5}^{n / 2} n^{1-k / 4} .
$$

Por outro lado, veja que

$$
\sum_{k=5}^{n / 2} n^{1-k / 4}=\sum_{k-4=1}^{n / 2}\left[n^{-1 / 4}\right]^{k-4}=\sum_{k-4=0}^{n / 2}\left[n^{-1 / 4}\right]^{k-4}-1,
$$

logo como $n^{-1 / 4}<1$, então a soma dos $m$ primeiros números de uma série geométrica é

$$
\sum_{k-4=0}^{m}\left[n^{-1 / 4}\right]^{k-4}=\frac{1-\left[n^{-1 / 4}\right]^{m+1}}{1-n^{-1 / 4}}=\frac{1-\left[\frac{1}{n^{1 / 4}}\right]^{n / 2+1}}{1-\frac{1}{n^{1 / 4}}}
$$

onde $m=n / 2$. Mas por 2.23 e 2.24 , temos

$$
\sum_{k=5}^{n / 2} n^{1-k / 4}=\frac{1-\left[\frac{1}{n^{1 / 4}}\right]^{n / 2+1}}{1-\frac{1}{n^{1 / 4}}}-1=o(1) .
$$

Portanto, por 2.25 e 2.22, concluímos que

$$
\mathbb{P}_{\lambda}\left(E R_{n}(\lambda / n) \text { desconectado, } Y=0\right) \leq o(1) .
$$

Isto prova a Proposição 2.2.2.

\subsubsection{Prova do Teorema 1.2.9}

Prova. Primeiro queremos mostrar que para $\lambda-\log n \rightarrow \infty$, a sequência de grafos $\left(E R_{n}\right)_{n \geq 1}$, é conectada com alta probabilidade, isto é $\mathbb{P}_{\lambda}\left(E R_{n}(\lambda / n)\right.$ conectado $) \rightarrow 1$. Para isto, observe que pela Proposição 2.2.2 temos

$$
\mathbb{P}_{\lambda}\left(E R_{n}(\lambda / n) \text { conectado }\right)=\mathbb{P}_{\lambda}(Y=0)+o(1),
$$

então com isto é suficiente mostrar que $\mathbb{P}_{\lambda}(Y=0) \rightarrow 1$ quando $\lambda-\log n \rightarrow \infty$. Então pela desigualdade de Markov, no Proposição 2.1.11, temos

$$
\mathbb{P}_{\lambda}(Y=0)=1-\mathbb{P}_{\lambda}(Y>0)=1-\mathbb{P}_{\lambda}(Y \geq 1) \geq 1-\mathbb{E}_{\lambda}[Y]
$$

Logo por (2.14) temos

$$
\mathbb{P}_{\lambda}(Y=0) \geq 1-n e^{-\lambda} e^{\lambda / n}=1-n e^{-\lambda} O(1)=1-e^{-(\lambda-\log n)} O(1) .
$$


Assim $\mathbb{P}_{\lambda}(Y=0) \rightarrow 1$ quando $\lambda-\log n \rightarrow \infty$ ou seja que o grafo aleatório Erdös-Rényi é conectado com alta probabilidade.

Por outro lado, também queremos mostrar que o grafo aleatório Erdös-Rényi é desconectado com alta probabilidade, para $\lambda-\log n \rightarrow-\infty$. Por (2.18), basta provar que $\mathbb{P}_{\lambda}(Y=0) \rightarrow 0$ quando $\lambda-\log n \rightarrow-\infty$, para isto, pela Proposição 2.2.1 temos que

$$
\mathbb{E}_{\lambda}[Y]=n e^{-\lambda}\left(1+O\left(\lambda^{2} / n\right)\right)=n e^{-\lambda}(1+o(1))=e^{-(\lambda-\log n)}(1+o(1)),
$$

então, $\mathbb{E}_{\lambda}[Y] \rightarrow \infty$ quando $\lambda-\log n \rightarrow-\infty$. Logo pela Desigualdade chebyshev, na Proposição 2.1.12, temos

$$
\mathbb{P}_{\lambda}(Y=0) \leq \frac{\operatorname{Var}_{\lambda}(Y)}{\mathbb{E}_{\lambda}[Y]^{2}} \leq \frac{\mathbb{E}_{\lambda}[Y]+\frac{\lambda}{n-\lambda} \mathbb{E}_{\lambda}[Y]^{2}}{\mathbb{E}_{\lambda}[Y]^{2}}=\frac{1}{\mathbb{E}_{\lambda}[Y]}+\frac{\lambda}{n-\lambda},
$$

portanto, pelo fato de que $\lambda \leq \log n$ e quando $\lambda-\log n \rightarrow-\infty$, então $\mathbb{P}_{\lambda}(Y=0) \rightarrow 0$, isto completa a prova de que $E R_{n}(\lambda / n)$ é desconectado com alta probabilidade para $\lambda-\log n \rightarrow-\infty$.

\subsection{Prova do Teorema da Janela crítica para a conec- tividade}

Antes de começar a prova, vejamos a seguinte proposição.

Proposição 2.3.1. Seja $Y$ a variável aleatória que representa o número de vértices isolados de $E R_{n}(\lambda / n)$, definido em (1.1). Y converge em distribuição para uma v. a $Z$ Poisson com parâmetro $e^{-t}$, para $\lambda=\log n+t, t \in \mathbb{R}$ fixo.

Prova. Sabemos que por $(2.13) \mathbb{E}_{\lambda}[Y]=n e^{-\lambda}\left(1+O\left(\lambda^{2} / n\right)\right)$, então

$$
\lim _{n \rightarrow \infty} \mathbb{E}_{\lambda}[Y]=e^{-t}
$$

Para demostrar a convergência em distribuição de $Y$ para $Z$ usaremos os Teoremas 2.1.8 e 2.1.9, é suficiente provar que

$$
\lim _{n \rightarrow \infty} \mathbb{E}\left[(Y)_{r}\right]=\lim _{n \rightarrow \infty} \sum_{i_{1}, \ldots, i_{r}}^{*} \mathbb{P}_{\lambda}\left(I_{i_{1}}=\ldots=I_{i_{r}}=1\right)=e^{t r}
$$

para todo $r \geq 1$, onde $I_{i}$ está definida em (1.1) e a soma varia sobre todos os $i_{1}, \ldots, i_{r} \in$ $\{1, \ldots, n\}$ que são distintos.

Pela permutabilidade dos vértices $\mathbb{P}_{\lambda}\left(I_{i_{1}}=\ldots=I_{i_{r}}=1\right)$ é independente da escolha exata dos índices $i_{1}, \ldots, i_{r}$, então podemos escrever

$$
\mathbb{P}_{\lambda}\left(I_{i_{1}}=\ldots=I_{i_{r}}=1\right)=\mathbb{P}_{\lambda}\left(I_{1}=\ldots=I_{r}=1\right) .
$$


Logo usando o fato de que existem $n(n-1) \ldots(n-r+1)$ distintas escolhas de $i_{1}, \ldots, i_{r} \in$ $\{1, \ldots, n\}$, podemos escrever

$$
\mathbb{E}_{\lambda}\left[(Y)_{r}\right]=n(n-1) \ldots(n-r+1) \mathbb{P}_{\lambda}\left(I_{1}=\ldots=I_{r}=1\right)=\frac{n !}{(n-r) !} \mathbb{P}_{\lambda}\left(I_{1}=\ldots=I_{r}=1\right) .
$$

Note que o evento $\left\{I_{1}=\ldots=I_{r}=1\right\}$ ocorre quando as st arestas estão vacantes, com $s \in\{1, \ldots, r\}$ e $t \in\{1, \ldots, n\}$, logo desde que estas arestas são todas independentes podemos escrever o seguinte

$$
\begin{aligned}
\mathbb{P}_{\lambda}\left(I_{1}=\ldots=I_{r}=1\right) & =\mathbb{P}_{\lambda}\left(I_{1}=1\right) \mathbb{P}_{\lambda}\left(I_{2}=1 \mid I_{1}=1\right) \cdots \mathbb{P}_{\lambda}\left(I_{r}=1 \mid I_{r-1}=1, \ldots, I_{1}=1\right) \\
& =\left(1-\frac{\lambda}{n}\right)^{n-1}\left(1-\frac{\lambda}{n}\right)^{n-2} \ldots\left(1-\frac{\lambda}{n}\right)^{n-r} \\
& =\left(1-\frac{\lambda}{n}\right)^{n r-r(r+1) / 2} \\
& =n^{-r}\left[n\left(1-\frac{\lambda}{n}\right)^{n-1}\right]^{r}\left(1-\frac{\lambda}{n}\right)^{-r(r-1) / 2} .
\end{aligned}
$$

Usando o fato de que $\mathbb{E}_{\lambda}[Y]=n\left(1-\frac{\lambda}{n}\right)^{n-1}$ e pelo Apêndice A3 obtemos

$$
\mathbb{P}_{\lambda}\left(I_{1}=\ldots=I_{r}=1\right)=n^{-r}\left(\mathbb{E}_{\lambda}[Y]\right)^{r}(1+o(1)) .
$$

Assim, aplicando limite em (2.27) obtemos

$$
\begin{aligned}
\lim _{n \rightarrow \infty} \mathbb{E}_{\lambda}\left[(Y)_{r}\right] & =\lim _{n \rightarrow \infty} \frac{n !}{(n-r) !} n^{-r} \mathbb{E}_{\lambda}[Y]^{r} \\
& =\lim _{n \rightarrow \infty} \frac{n(n-1) \ldots(n-r+1)}{n^{r}} \mathbb{E}_{\lambda}[Y]^{r}
\end{aligned}
$$

Por fim por (2.26) temos

$$
\lim _{n \rightarrow \infty} \mathbb{E}_{\lambda}\left[(Y)_{r}\right]=e^{-t r}
$$

com isto provamos que $Y \stackrel{d}{\rightarrow} Z$.

\subsubsection{Prova do Teorema 1.3.1}

Prova. Pela convergência em distribuição de $Y$ para uma v.a $Z \sim \operatorname{Poisson}\left(e^{-t}\right)$, na Proposição 2.3.1, podemos escrever

$$
\mathbb{P}_{\lambda}(Y=0)=\mathbb{P}_{\lambda}(Z=0)+o(1)=e^{-e^{-t}}+o(1) .
$$

Logo pela Proposição 2.2.2, temos

$$
\mathbb{P}_{\lambda}\left(E R_{n}(\lambda / n) \text { conectado }\right)=e^{-e^{-t}}+o(1)=e^{-e^{-t}}(1+o(1)),
$$

para $\lambda=\log n+t \rightarrow \infty$ e para algum $t \in \mathbb{R}$ fixo. A ultima igualdade pelo Apêndice A3 4 e desde que $t$ é constante. 


\section{Capítulo 3}

\section{Grafo aleatório com topologia simples}

O grafo aleatório Erdös-Rényi não tem topologia. Neste capítulo estudamos o número de vértices isolados para uma extensão do modelo Erdös-Rényi, em que é adicionado uma topologia simples. Nesta seção estudamos um comportamento da variável de interesse, para o novo modelo apresentado na seguinte seção. Por fim provaremos a convergência do número de vértices isolados para uma Poisson.

\subsection{Descrição do modelo}

Denotamos por $G_{n}(p, q, 1)$ o grafo aleatório que pode ser construído da seguinte forma. Considere o conjunto de vértices $V=\left\{v_{1}, \ldots, v_{n}\right\}$ localizados sobre um anel. Cada vértice está rodeado por exatamente dois vizinhos mais próximos de distância 1 (um à esquerda e um à direita). Considere a distância entre dois $v_{i}$ e $v_{j}$, definida por

$$
d\left(v_{i}, v_{j}\right)=\min \{|j-i|, n-|j-i|\} .
$$

Cada par de vértices $v_{i}$ e $v_{j}$ é conectados por uma aresta com probabilidade $p_{n}$ de forma independente dos outros, $p_{n}=\lambda / n$, tal como no modelo Erdös-Rényi. Independentemente das conexões com probabilidade $p$, os vértices deste grafo podem ser conectados aos seus vizinhos mais próximos, de distância 1 , com uma probabilidade $q$, isto é, pode-se conectar o vértice $v_{i}$ com seus vizinhos mais próximos $v_{i-1}$ e $v_{i+1}$, com probabilidade $q$, onde $q$ depende de $n$, mas por simplicidade não vamos considerar $n$ na notação, ver Fig. 3.1. Neste modelo supomos que tanto $v_{i}$ pode se conectar com $v_{i+1}$, como $v_{i+1}$ com $v_{i}$ de forma independente. Logo, denotemos por $\mathbb{P}_{\lambda, q}[i \leftrightarrow j]$ a probabilidade de que os vértices $v_{i}$ e $v_{j}$ estejam conectados, isto é

$$
\mathbb{P}_{\lambda, q}[i \leftrightarrow j]=\left\{\begin{array}{l}
p \quad, \quad \text { se } d\left(v_{i}, v_{j}\right)>1 \\
1-(1-p)(1-q)^{2}, \text { se } d\left(v_{i}, v_{j}\right)=1
\end{array}\right.
$$

Nas seções anteriores foi estudado o limiar da conectividade sobre $\lambda$ considerando o modelo Erdös-Rényi, e vimos como esta propriedade depende do número de vértices isolados. 


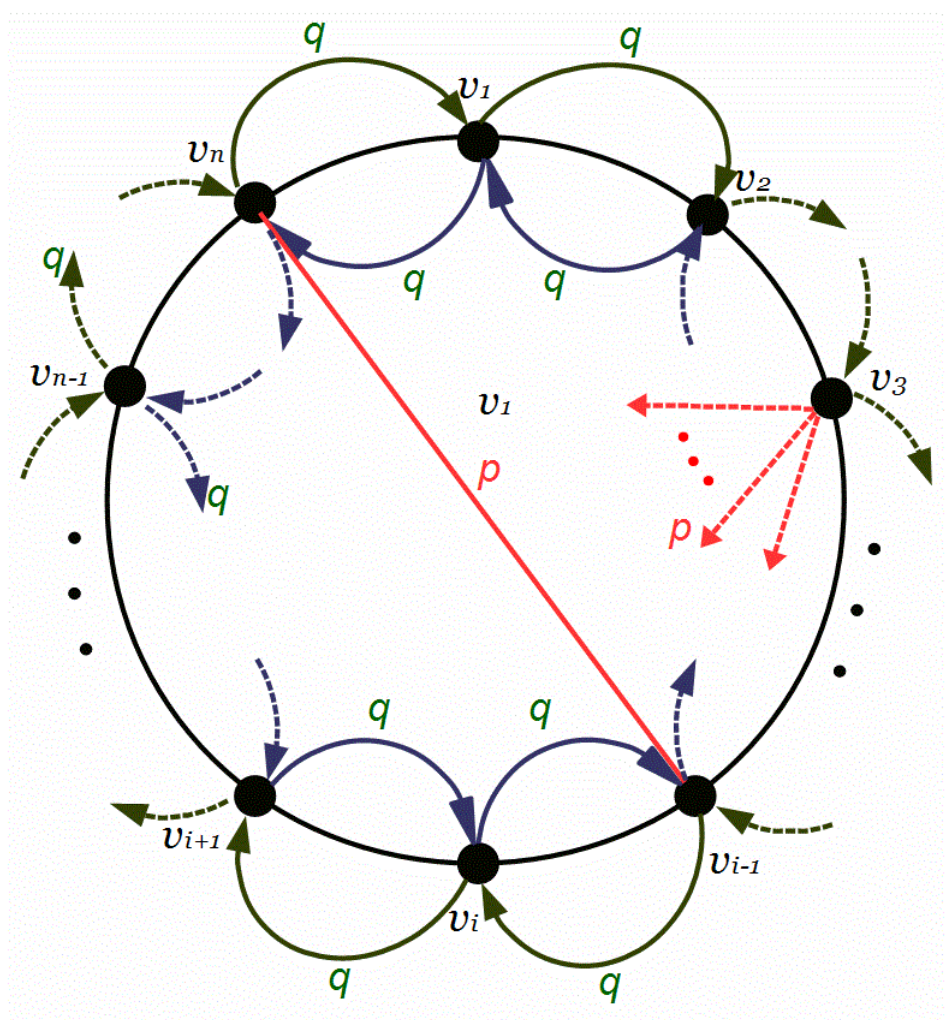

Figura 3.1: Representação gráfica de $G_{n}(p, q, 1)$.

Se consideramos o modelo acrescentando uma topologia, descrito acima, seria interessante estudar o comportamento do número de vértices isolados dependendo de $\lambda$ e a probabilidade $q$. Note que a probabilidade $q$ é um parâmetro que só pode ajuda a conectar o grafo. Portanto, quando $q \rightarrow 0$, espera-se que o grafo fique simplesmente como o grafo aleatório Erdös-Rényi, (ver Observação 3.1.4). Por outro lado, quando $q \rightarrow 1$, ele facilita a conectividade do grafo (Ver exemplo 3.1.3).

Da mesma forma como o caso do modelo Erdös-Rényi, definimos o número de vértices isolados do grafo $G_{n}(p, q, 1)$, pela v.a. $Y^{\prime}$ como

$$
Y^{\prime}=\sum_{i=1}^{n} I_{i}^{\prime}, \quad \text { onde } \quad I_{i}^{\prime}=\mathbb{1}_{\{|\mathcal{C}(i)|=1\}} .
$$

Em que $|\mathcal{C}(i)|=1$, quer dizer que o vértice $i$ não é conectado com nenhum outro vértice. Neste capítulo provamos o seguinte resultado

Teorema 3.1.1. Seja $G_{n}(p, q, 1)$, com $p=\lambda / n$.

(a) Se $\lambda-\log \left[(1-q)^{4} n\right] \rightarrow-\infty$, então $\mathbb{P}_{\lambda, q}\left[Y^{\prime}=0\right] \rightarrow 0$.

(b) Se $\lambda-\log \left[(1-q)^{4} n\right] \rightarrow \infty$, então $\mathbb{P}_{\lambda, q}\left[Y^{\prime}=0\right] \rightarrow 1$. 
(c) Se $\lambda-\log \left[(1-q)^{4} n\right]=t$, com $t \in \mathbb{R}$, então $\mathbb{P}_{\lambda, q}\left[Y^{\prime}=0\right] \rightarrow e^{-e^{-t}}$.

\section{Observação 3.1.2.}

1. O fato de que a probabilidade de não ter vértices isolados vai para 0 , quer dizer que o grafo vai ter pelo menos um vértice isolado, então isto é suficiente para considerar o grafo como desconectado. Do item $(a)$ no Teorema 3.1.1, podemos ver que, se o valor de $\lambda$ for muito menor do que $\log \left[(1-q)^{4} n\right]$ o número de vértices isolados no grafo $G_{n}(p, q, 1)$ vai-se acrescentar quando levamos o $n$ para o infinito. Então, se falamos em termos probabilísticos, com a mesma condição, a probabilidade de não ter vértices isolados no grafo vai ter que ir para 0.

2. Por outro lado, se a probabilidade de não ter vértices isolados vai para 1, só garante a não existência de vértices isolados, quando $n \rightarrow \infty$. Isto não é suficiente para garantir que o grafo seja completamente conectado, pois pode-se dar o caso em que o grafo tenha por exemplo dois componentes conectadas. Neste caso veja que não existem vértices isolados mas o grafo não é conectado. O item (b) no Teorema 3.1.1 explica que se $\lambda$ for muito maior do que $\log \left[(1-q)^{4} n\right]$, então o número de vértices isolados vai decair para 0 . Veja que, embora este resultado não garante a conectividade do grafo $G_{n}(p, q, 1)$, ele pode significar uma ajuda importante para conectar o grafo assintóticamente, sempre que $\lambda-\log \left[(1-q)^{4} n\right] \rightarrow \infty$. No exemplo 3.1.3, temos possíveis valores de $\lambda$ e $q$ dependentes de $n$, de tal forma que $\lambda-\log \left[n(1-q)^{4}\right] \rightarrow \infty$.

Exemplo 3.1.3. Um exemplo interessante seria encontrar $\lambda$ e $q$ de tal forma que, com este $\lambda$, no caso do modelo Erdös-Rényi, a média do número de vértices isolados vai para infinito, enquanto no grafo $G_{n}(p, q, 1)$, ele vai para o 0 , pois o parâmetro $q$ deve ajudar a conectar o grafo. Para isto vamos considerar $\lambda=\alpha \log n$, com $\alpha \in(0,1)$, e $q=1-n^{-\beta}$, com $\beta>0$. Assim, quando $\alpha<1$, sabemos que no caso do modelo Erdös-Rényi, o numero esperado de vértices isolados vai para infinito. Então dependendo do valor de $\beta$, o parâmetro $q$ pode ajudar a conectar o grafo. Para isto, precisamos que $\lambda-\log \left[n(1-q)^{4}\right] \rightarrow \infty, \operatorname{logo}$ considerando os valores de $\lambda$ e $q$, mencionados acima, temos que

$$
\begin{aligned}
\lambda-\log \left[n(1-q)^{4}\right] & =\alpha \log n-\log \left[n\left(\frac{1}{n}\right)^{4 \beta}\right] \\
& =\alpha \log n-\log n-4 \beta \log \frac{1}{n} \\
& =(1-\alpha) \log n-4 \beta \log n,
\end{aligned}
$$

então, para que $\lambda-\log \left[n(1-q)^{4}\right] \rightarrow \infty$ basta considerar $4 \beta>1-\alpha$, e assim podemos ajudar a conectar o grafo $G_{n}(p, q, 1)$.

Observação 3.1.4. Observe que se $q \rightarrow 0$, quando $n \rightarrow \infty$, este modelo fica como o modelos Erdös-Rényi. Por exemplo, $q=\frac{1}{n} \rightarrow 0$, quando $n \rightarrow \infty$, então veja que $\lambda-\log \left[n\left(1-\frac{1}{n}\right)^{4}\right] \rightarrow \infty$ é equivalente a $\lambda-\log n \rightarrow \infty$, quando $n \rightarrow \infty$, ou $\lambda-$ $\log \left[n\left(1-\frac{1}{n}\right)^{4}\right] \rightarrow-\infty$ é equivalente a $\lambda-\log n \rightarrow-\infty$, quando $n \rightarrow \infty$. 


\subsection{Esperança e variância de vértices isolados}

O principal resultado nesta seção é o Lema 3.2.1, cuja prova implica encontrar a esperança do número de vértices isolados e uma limitante para a variância.

Lema 3.2.1. Para todo $1 / 2 \leq \lambda \leq n / 2$,

$$
\mathbb{E}_{\lambda, q}\left[Y^{\prime}\right]=n(1-q)^{4} e^{-\lambda}\left(1+O\left(\lambda^{2} / n\right)\right)
$$

e para todo $\lambda \leq n$

$$
\operatorname{Var}_{\lambda, q}\left(Y^{\prime}\right) \leq\left[2(1-q)^{2}\left(1-\frac{\lambda}{n}\right)^{n-2}+1\right] \mathbb{E}_{\lambda, q}\left[Y^{\prime}\right]+\frac{\lambda}{n-\lambda} \mathbb{E}_{\lambda, q}\left[Y^{\prime}\right]^{2} .
$$

Prova. Note que o valor esperado de $Y^{\prime}$ é dado por

$$
\mathbb{E}_{\lambda, q}\left[Y^{\prime}\right]=\mathbb{E}_{\lambda, q}\left[\sum_{i=1}^{n} I_{i}^{\prime}\right]=n \mathbb{P}_{\lambda, q}[|\mathcal{C}(1)|=1]
$$

Seja $[i \leftrightarrow j]:=[i \leftrightarrow j]^{c}$, então

$$
\mathbb{P}_{\lambda, q}[|\mathcal{C}(1)|=1]=\prod_{i=2}^{n} \mathbb{P}_{\lambda, q}[1 \leftrightarrow j]=\mathbb{P}_{\lambda, q}[1 \leftrightarrow 2] \mathbb{P}_{\lambda, q}[1 \leftrightarrow n] \prod_{j=3}^{n-1} \mathbb{P}_{\lambda, q}[1 \leftrightarrow j],
$$

dado que os eventos $[1 \leftrightarrow j]$ são independentes para todo $j . \operatorname{Logo} \mathbb{P}_{\lambda, q}[|\mathcal{C}(1)|=1]=$ $(1-q)^{4}(1-p)^{n-1}$. Portanto,

$$
\mathbb{E}_{\lambda, q}\left[Y^{\prime}\right]=n(1-q)^{4}(1-p)^{n-1} .
$$

Usando que $x=e^{\log (x)}$, e a expansão de Taylor de $\log (1-p)$, note que $1-p=e^{\left(-p+O\left(p^{2}\right)\right)}$. Daí observe que $(1-p)^{n}=e^{-n p}\left(1+O\left(n p^{2}\right)\right)$, onde usamos a expansão de Taylor da exponencial, do segundo fator. Logo para $p=\lambda / n$, e por (3.7) obtemos que

$$
\mathbb{E}_{\lambda, q}\left[Y^{\prime}\right]=n(1-q)^{4} e^{-\lambda}\left(1+O\left(\lambda^{2} / n\right)\right),
$$

Com isto provamos (3.3). Este ultimo resultado pode ser escrito como

$$
\mathbb{E}_{\lambda, q}\left[Y^{\prime}\right]=e^{-\left(\lambda-\log \left[n(1-q)^{4}\right]\right)}\left(1+O\left(\lambda^{2} / n\right)\right) .
$$

Para provar (3.4) precisamos encontrar a variância, para isto sabemos que $\operatorname{Var}_{\lambda, q}\left(Y^{\prime}\right)=$ $\mathbb{E}_{\lambda, q}\left[Y^{\prime 2}\right]-\mathbb{E}_{\lambda, q}\left[Y^{\prime}\right]^{2}$, veja que a dificuldade aqui é calcular $\mathbb{E}_{\lambda, q}\left[Y^{\prime 2}\right]$. Note que podemos escrever o segundo momento fatorial de $Y^{\prime}$ como $\mathbb{E}_{\lambda, q}\left[\left(Y^{\prime}\right)_{2}\right]=\mathbb{E}_{\lambda, q}\left[Y^{\prime 2}\right]-\mathbb{E}_{\lambda, q}\left[Y^{\prime}\right]$. Então agora precisamos obter o valor de $\mathbb{E}_{\lambda, q}\left[\left(Y^{\prime}\right)_{2}\right]$ para o qual usaremos o Teorema 2.1.9, isto é

$$
\mathbb{E}_{\lambda, q}\left[\left(Y^{\prime}\right)_{2}\right]=\sum_{i_{1}, i_{2}}^{*} \mathbb{P}_{\lambda, q}\left[I_{i_{1}}^{\prime}=I_{i_{2}}^{\prime}=1\right]
$$

Note que $\mathbb{P}_{\lambda, q}\left[I_{i_{1}}^{\prime}=I_{i_{2}}^{\prime}=1\right]$ é a probabilidade de que dois vértices distintos do grafo são isolados. Faremos a escolha destes vértices em duas formas. Primeiro é que estes vértices 
sejam vizinhos, neste caso teria-se $2 n$ possíveis pares de vértices vizinhos. Segundo que estes dois vértices não sejam vizinhos que tem $n(n-3)$ formas de escolher. Com isto e usando a definição (3.2), temos que

$$
\begin{aligned}
\mathbb{E}_{\lambda, q}\left[\left(Y^{\prime}\right)_{2}\right] & =2 n \mathbb{P}_{\lambda, q}\left[I_{1}^{\prime}=I_{2}^{\prime}=1\right]+n(n-3) \mathbb{P}_{\lambda, q}\left[I_{1}^{\prime}=I_{3}^{\prime}=1\right] \\
& =2 n \mathbb{P}_{\lambda, q}[|\mathcal{C}(1)|=1,|\mathcal{C}(2)|=1]+n(n-3) \mathbb{P}_{\lambda, q}[|\mathcal{C}(1)|=1,|\mathcal{C}(3)|=1] \\
& =2 n \mathbb{P}_{\lambda, q}[|\mathcal{C}(1)|=1] \mathbb{P}_{\lambda, q}[|\mathcal{C}(2)|=1|| \mathcal{C}(1) \mid=1] \\
& +n(n-3) \mathbb{P}_{\lambda, q}[|\mathcal{C}(1)|=1] \mathbb{P}_{\lambda, q}[|\mathcal{C}(3)|=1|| \mathcal{C}(1) \mid=1] \\
& =2 n \mathbb{P}_{\lambda, q}[|\mathcal{C}(1)|=1](1-q)^{2}(1-p)^{n-2}+n(n-3) \mathbb{P}_{\lambda, q}[|\mathcal{C}(1)|=1](1-q)^{4}(1-p)^{n-2},
\end{aligned}
$$

logo por (3.5) e para $p=\lambda / n$, temos que

$$
\mathbb{E}_{\lambda, q}\left[\left(Y^{\prime}\right)_{2}\right]=2(1-q)^{2}(1-\lambda / n)^{n-2} \mathbb{E}_{\lambda, q}\left[Y^{\prime}\right]+(n-3)(1-q)^{4}(1-\lambda / n)^{n-2} \mathbb{E}_{\lambda, q}\left[Y^{\prime}\right],
$$

mas por (3.7), é equivalente a escrever,

$$
\mathbb{E}_{\lambda, q}\left[\left(Y^{\prime}\right)_{2}\right]=2(1-q)^{2}\left(1-\frac{\lambda}{n}\right)^{n-2} \mathbb{E}_{\lambda, q}\left[Y^{\prime}\right]+\left(\frac{n-3}{n-\lambda}\right) \mathbb{E}_{\lambda, q}\left[Y^{\prime}\right]^{2}
$$

Por outro lado, desde que $\left(Y^{\prime}\right)_{2}=Y^{\prime}\left(Y^{\prime}-1\right)$, então

$$
\mathbb{E}_{\lambda, q}\left[\left(Y^{\prime}\right)_{2}\right]=\mathbb{E}_{\lambda, q}\left[Y^{\prime 2}\right]-\mathbb{E}_{\lambda, q}\left[Y^{\prime}\right],
$$

portanto como $\operatorname{Var}_{\lambda, q}\left(Y^{\prime}\right)=\mathbb{E}_{\lambda, q}\left[Y^{\prime 2}\right]-\mathbb{E}_{\lambda, q}\left[Y^{\prime}\right]^{2}$ e por (3.10) e (3.11), obtemos

$$
\begin{aligned}
\operatorname{Var}_{\lambda, q}\left(Y^{\prime}\right) & =\left[2(1-q)^{2}\left(1-\frac{\lambda}{n}\right)^{n-2}+1\right] \mathbb{E}_{\lambda, q}\left[Y^{\prime}\right]+\left[\frac{n-3}{n-\lambda}-1\right] \mathbb{E}_{\lambda, q}\left[Y^{\prime}\right]^{2} \\
& \leq\left[2(1-q)^{2}\left(1-\frac{\lambda}{n}\right)^{n-2}+1\right] \mathbb{E}_{\lambda, q}\left[Y^{\prime}\right]+\frac{\lambda}{n-\lambda} \mathbb{E}_{\lambda, q}\left[Y^{\prime}\right]^{2}
\end{aligned}
$$

Com isto provamos o resultado (3.4), portanto também o Lema 3.2.1.

\section{Proposição 3.2.2.}

(a) $\operatorname{Se} \lambda-\log \left[n(1-q)^{4}\right] \rightarrow-\infty$ então $\mathbb{E}_{\lambda, q}\left[Y^{\prime}\right] \rightarrow \infty$.

(b) Se $\lambda-\log \left[n(1-q)^{4}\right] \rightarrow \infty$ então $\mathbb{E}_{\lambda, q}\left[Y^{\prime}\right] \rightarrow 0$.

Prova. Para provar o item (a) note que por (3.9) temos

$$
\mathbb{E}_{\lambda, q}\left[Y^{\prime}\right]=e^{-\left(\lambda-\log \left[n(1-q)^{4}\right]\right)}\left(1+O\left(\lambda^{2} / n\right)\right),
$$

com isto é fácil ver que $\mathbb{E}_{\lambda, q}\left[Y^{\prime}\right] \rightarrow \infty$ quando $\lambda-\log \left[n(1-q)^{4}\right] \rightarrow-\infty$, isto prova o item (a), de forma similar para o item (b). 


\subsection{Distribuição dos vértices isolados}

O objetivo desta seção é mostrar que a v.a. $Y^{\prime}$ converge em distribuição para uma v.a. $Z \sim \operatorname{Poisson}\left(e^{-t}\right)$. Para isto vejamos a seguinte proposição.

Proposição 3.3.1. Seja a v.a. $Y^{\prime}$, número de vértices isolados, definida em (3.2), e a v.a. Z com distribuição Poisson $\left(\lim _{n \rightarrow \infty} \mathbb{E}_{\lambda, q}\left[Y^{\prime}\right]\right)$. Quando $\lambda=\log \left[(1-q)^{4} n\right]+t$, para $t \in \mathbb{R}$ fixo,

$$
Y^{\prime} \stackrel{d}{\rightarrow} Z
$$

Prova. Veja que por (3.9), $\mathbb{E}_{\lambda, q}\left[Y^{\prime}\right]=e^{-\left(\lambda-\log \left[n(1-q)^{4}\right]\right)}\left(1+O\left(\lambda^{2} / n\right)\right)$, e desde que $\lambda=$ $\log \left[(1-q)^{4} n\right]+t, t$ fixo, então

$$
\lim _{n \rightarrow \infty} \mathbb{E}_{\lambda, q}\left[Y^{\prime}\right]=e^{-t}
$$

com isto e pelo Teorema 2.1.8 (Convergência para uma variável aleatória Poisson) basta mostrar que

$$
\lim _{n \rightarrow \infty} \mathbb{E}_{\lambda, q}\left[\left(Y^{\prime}\right)_{r}\right]=e^{-t r}
$$

Por outro lado pelo Teorema 2.1.9 sabemos que

$$
\mathbb{E}\left[\left(Y^{\prime}\right)_{r}\right]=\sum_{i_{1}, \ldots, i_{r}}^{*} \mathbb{P}_{\lambda, q}\left(I_{i_{1}}^{\prime}=\ldots=I_{i_{r}}^{\prime}=1\right),
$$

com $\sum_{i_{1}, \ldots, i_{r}}^{*}$ que denota uma soma em índices distintos, para todo $r \geq 1$, onde $I_{i}^{\prime}$ está definida em (3.2). Note que $\mathbb{P}_{\lambda, q}\left(I_{i_{1}}^{\prime}=\ldots=I_{i_{r}}^{\prime}=1\right)$ é a probabilidade de que o grafo de tamanho $n$, tenha $r$ vértices isolados, com $n$ suficientemente grande, e $\sum_{i_{1}, \ldots, i_{r}}^{*}$ é a quantidade total de formas possíveis de escolher $r$ vértices dos $n$ vértices.

Observe que neste modelo temos dois tipos de conexões independentes, que acontecem com probabilidade $p$ e $q$. Logo podemos escrever

$$
\mathbb{P}_{\lambda, q}\left(I_{i_{1}}^{\prime}=\ldots=I_{i_{r}}^{\prime}=1\right)=\mathbb{P}_{q}\left(I_{i_{1}}^{\prime}=\ldots=I_{i_{r}}^{\prime}=1 \mid I_{i_{1}}=\ldots=I_{i_{r}}=1\right) \mathbb{P}_{\lambda}\left(I_{i_{1}}=\ldots=I_{i_{r}}=1\right),
$$

onde $Y=\sum_{i=1}^{r} I_{i}$, é o número de vértices isolados, considerando apenas o grafo aleatório Erdös-Rényi $E R_{n}(\lambda / n), \operatorname{com} \lambda=\log \left[(1-q)^{4} n\right]+t$. Logo veja que por (2.28)

$$
\mathbb{P}_{\lambda}\left(I_{i_{1}}=\ldots=I_{i_{r}}=1\right)=n^{-r}\left(\mathbb{E}_{\lambda}[Y]\right)^{r}(1+o(1)) .
$$

Então,

$$
\mathbb{E}\left[\left(Y^{\prime}\right)_{r}\right]=\left[\sum_{i_{1}, \ldots, i_{r}}^{*} \mathbb{P}_{q}\left(I_{i_{1}}^{\prime}=\ldots=I_{i_{r}}^{\prime}=1 \mid I_{i_{1}}=\ldots=I_{i_{r}}=1\right)\right] n^{-r}\left(\mathbb{E}_{\lambda}[Y]\right)^{r}(1+o(1)),
$$

mas sabemos que $\mathbb{E}_{\lambda}[Y]=n e^{-\lambda}\left(1+O\left(\lambda^{2} / n\right)\right)$, por (2.12), na Proposição 2.2.1. Logo para $\lambda=\log \left[(1-q)^{4} n\right]+t$, temos que

$$
\mathbb{E}\left[\left(Y^{\prime}\right)_{r}\right]=\frac{e^{-t r}}{(1-q)^{4 r} n^{r}}(1+o(1)) \sum_{i_{1}, \ldots, i_{r}}^{*} \mathbb{P}_{q}\left(I_{i_{1}}^{\prime}=\ldots=I_{i_{r}}^{\prime}=1 \mid I_{i_{1}}=\ldots=I_{i_{r}}=1\right) .
$$


Então devemos mostrar que

$$
\frac{1}{(1-q)^{4 r} n^{r}} \sum_{i_{1}, \ldots, i_{r}}^{*} \mathbb{P}_{q}\left(I_{i_{1}}^{\prime}=\ldots=I_{i_{r}}^{\prime}=1 \mid I_{i_{1}}=\ldots=I_{i_{r}}=1\right)=(1+o(1)) .
$$

Logo, para calcular $\mathbb{P}_{q}\left(I_{i_{1}}^{\prime}=\ldots=I_{i_{r}}^{\prime}=1 \mid I_{i_{1}}=\ldots=I_{i_{r}}=1\right)$ somente consideramos as conexões relacionadas à probabilidade $q$. Vamos denotar esta probabilidade como $p\left(i_{1}, \ldots, i_{r}\right)$, então para calcular

$$
\sum_{i_{1}, \ldots, i_{r}}^{*} p\left(i_{1}, \ldots, i_{r}\right)
$$

vamos separar a soma em duas partes

1.

$$
\sum_{i_{1}, \ldots, i_{r} \in A}^{*} p\left(i_{1}, \ldots, i_{r}\right),
$$

onde $A$ é o conjunto dos $r$ vértices escolhidos de tal forma que eles não sejam vizinhos. Então precisamos encontrar a quantidade de todas as formas possíveis de escolher $r$ vértices do grafo, de modo que não sejam vizinhos, esta quantidade é o tamanho de $A$ denotada por $|A|$. Então usando o segundo lema de Kaplansky (ver Apêndice A2.4 podemos obter a combinação de $r$ vértices não vizinhos, dos $n$ vértices localizados num anel, que é dado por $\frac{n}{n-r}\left(\begin{array}{c}n-r \\ r\end{array}\right)$. Mas ainda devemos considerar a quantidade de formas de ordenar estos $r$ vértices, que é um problema de permutação simples, e é dado por $r$ ! (ver Apêndice A2.1). Então temos

$$
|A|=r ! \frac{n}{n-r}\left(\begin{array}{c}
n-r \\
r
\end{array}\right)
$$

Logo

$$
|A|=\frac{n}{n-r} \frac{(n-r) !}{(n-2 r) !}=n(n-r-1) \cdots(n-2 r+1) .
$$

Por outro lado, veja que

$$
p\left(i_{1}, \ldots, i_{r}\right)=\mathbb{P}_{q}\left(I_{i_{1}}^{\prime}=1\right) \mathbb{P}_{q}\left(I_{i_{2}}^{\prime}=1\right) \ldots \mathbb{P}_{q}\left(I_{i_{r}}^{\prime}=1\right)=\left[(1-q)^{4}\right]^{r},
$$

desde que os $r$ vértice escolhidos não são vizinhos, neste caso as v.a's $I_{j}^{\prime}$ são independentes. Com isto temos que

$$
\sum_{i_{1}, \ldots, i_{r} \in A}^{*} p\left(i_{1}, \ldots, i_{r}\right)=(1-q)^{4 r} n(n-r-1) \cdots(n-2 r+1)
$$

então

$$
\frac{1}{(1-q)^{4 r} n^{r}} \sum_{i_{1}, \ldots, i_{r} \in A}^{*} p\left(i_{1}, \ldots, i_{r}\right)=\frac{n(n-r-1) \cdots(n-2 r+1)}{n^{r}}
$$

observe que o produto $n(n-r-1) \cdots(n-2 r+1)$ tem termos de números consecutivos que decrescem, então podemos dizer que este produto tem $1(n-r-1)-(n-2 r+$ 1) $+1=r-1$ termos, logo 


$$
\frac{n(n-r-1) \cdots(n-2 r+1)}{n^{r}}=(1+o(1))
$$

então

$$
\frac{n^{-r}}{(1-q)^{4 r}} \sum_{i_{1}, \ldots, i_{r} \in A}^{*} p\left(i_{1}, \ldots, i_{r}\right)=(1+o(1)) .
$$

Isto quer dizer que para provar (3.16), devemos mostrar que a outra parte da soma é $o(1)$.

2. Note que

$$
\frac{1}{(1-q)^{4 r} n^{r}} \sum_{i_{1}, \ldots, i_{r} \in A^{c}}^{*} p\left(i_{1}, \ldots, i_{r}\right) \leq \frac{\left|A^{c}\right|}{(1-q)^{4 r} n^{r}}
$$

onde $\left|A^{c}\right|$ é a quantidade de todas as formas possíveis de escolher $r$ vértices dos $n$ vértices localizados num anel, de modo que pelo menos 2 vértices sejam vizinhos. Então temos

$$
\frac{\left|A^{c}\right|}{n^{r}}=\frac{r !\left(\begin{array}{l}
n \\
r
\end{array}\right)-|A|}{n^{r}}=\frac{1}{n^{r}} \frac{n !}{(n-r) !}-\frac{|A|}{n^{r}} .
$$

Veja que

$$
\frac{|A|}{n^{r}}=\frac{r ! \frac{n}{n-r}\left(\begin{array}{c}
n-r \\
r
\end{array}\right)}{n^{r}}=(1+o(1))
$$

e também

$$
\frac{1}{n^{r}} \frac{n !}{(n-r) !}=\frac{n(n-1) \cdots(n-r+1)}{n^{r}}=(1+o(1))
$$

Então

$$
\frac{\left|A^{c}\right|}{n^{r}}=o(1)
$$

Portanto por (3.17) e (3.18), temos (3.16). Então $\mathbb{E}_{\lambda, q}\left[\left(Y^{\prime}\right)_{r}\right]=\left(1+(o(1)) e^{-t r}\right.$, assim

$$
\lim _{n \rightarrow \infty} \mathbb{E}_{\lambda, q}\left[\left(Y^{\prime}\right)_{r}\right]=e^{-t r} .
$$

Então concluímos que a v.a. $Y^{\prime}$ converge em distribuição para a v.a. $Z \sim \operatorname{Poisson}\left(e^{-t}\right)$, onde $e^{-t}=\lim _{n \rightarrow \infty} \mathbb{E}_{\lambda, q}\left[Y^{\prime}\right]$, e $\lambda=\log \left[(1-q)^{4} n\right]+t, t \in \mathbb{R}$ fixo. 


\subsection{Prova do Teorema 3.1.1}

Para provar o Teorema 3.1.1, usaremos os resultados das Proposições 3.2.2 e 3.3.1.

Prova. (a) Devemos mostrar que, se $\lambda-\log \left[(1-q)^{4} n\right] \rightarrow-\infty$, então $\mathbb{P}_{\lambda, q}\left(Y^{\prime}=0\right) \rightarrow 0$. Pela desigualdade de Chebyshev na Proposição 2.1 .12 e por (3.13) temos

$$
\begin{aligned}
\mathbb{P}_{\lambda, q}\left[Y^{\prime}=0\right] & \leq \frac{\operatorname{Var}_{\lambda, q}\left(Y^{\prime}\right)}{\mathbb{E}_{\lambda, q}\left[Y^{\prime}\right]^{2}} \\
& \leq \frac{\left[2(1-q)^{2}\left(1-\frac{\lambda}{n}\right)^{n-2}+1\right] \mathbb{E}_{\lambda, q}\left[Y^{\prime}\right]+\frac{\lambda}{n-\lambda} \mathbb{E}_{\lambda, q}\left[Y^{\prime}\right]^{2}}{\mathbb{E}_{\lambda, q}\left[Y^{\prime}\right]^{2}} \\
& \leq \frac{2(1-q)^{2}\left(1-\frac{\lambda}{n}\right)^{n-2}}{\mathbb{E}_{\lambda, q}\left[Y^{\prime}\right]}+\frac{\lambda}{n-\lambda}
\end{aligned}
$$

Pelo item (a), na Proposição 3.2.2, temos que se $\lambda-\log \left[n(1-q)^{4}\right] \rightarrow-\infty$ então $\mathbb{E}_{\lambda, q}\left[Y^{\prime}\right] \rightarrow \infty$, com isto temos que

$$
\frac{2(1-q)^{2}\left(1-\frac{\lambda}{n}\right)^{n-2}}{\mathbb{E}_{\lambda, q}\left[Y^{\prime}\right]} \rightarrow 0,
$$

quando $\lambda-\log \left[n(1-q)^{4}\right] \rightarrow-\infty$, desde que o numerador é limitado e $q, p<1$ com $p=\lambda / n$. Logo pelo fato de que $\lambda \leq \log \left[n(1-q)^{4}\right]$, quando $\lambda-\log \left[n(1-q)^{4}\right] \rightarrow-\infty$, então

$$
\frac{\lambda}{n-\lambda} \leq \frac{\log \left[n(1-q)^{4}\right]}{n-\log \left[n(1-q)^{4}\right]} \rightarrow 0
$$

em particular quando $\log \left[n(1-q)^{4}\right] \rightarrow \infty$. Portanto $\mathbb{P}_{\lambda, q}\left[Y^{\prime}=0\right] \rightarrow 0$ quando $\lambda-\log \left[n(1-q)^{4}\right] \rightarrow-\infty$.

(b) Devemos mostrar que $\mathbb{P}_{\lambda, q}\left(Y^{\prime}=0\right) \rightarrow 1$ quando $\lambda-\log \left[(1-q)^{4} n\right] \rightarrow \infty$. Então pela desigualdade de Markov, na Proposição 2.1.11, temos

$$
\mathbb{P}_{\lambda, q}\left(Y^{\prime}=0\right)=1-\mathbb{P}_{\lambda, q}\left(Y^{\prime} \geq 1\right)=\geq 1-\mathbb{E}_{\lambda, q}\left[Y^{\prime}\right]
$$

Logo por (2.14) temos

$\mathbb{P}_{\lambda, q}\left(Y^{\prime}=0\right) \geq 1-n(1-q)^{4} e^{-\lambda} e^{\lambda / n}=1-n(1-q)^{4} e^{-\lambda} O(1)=1-e^{-\left(\lambda-\log \left[(1-q)^{4} n\right]\right)} O(1)$.

$\operatorname{Assim} \mathbb{P}_{\lambda, q}\left(Y^{\prime}=0\right) \rightarrow 1$ quando $\lambda-\log \left[(1-q)^{4} n\right] \rightarrow \infty$ 
(c) Devemos mostrar que $\mathbb{P}_{\lambda, q}\left(Y^{\prime}=0\right) \rightarrow e^{-e^{-t}}$, quando $\lambda-\log \left[(1-q)^{4} n\right]=t$, com $t \in \mathbb{R}$. Então pela Proposição 3.3.1, sabemos que $Y^{\prime}$ converge em distribuição para uma v.a. Z Poisson $\left(e^{-t}\right)$, quando $\lambda=\log \left[(1-q)^{4} n\right]+t$ e $t \in \mathbb{R}$. Então temos que $\mathbb{P}_{\lambda, q}(Z=0)=e^{-e^{-t}}$, portanto

$$
\mathbb{P}_{\lambda, q}\left(Y^{\prime}=0\right) \rightarrow e^{e^{-t}}
$$

Com isto concluirmos a prova do Teorema 3.1.1. 


\section{Capítulo 4}

\section{Conclusão}

O objetivo desta pesquisa é estudar a limiar da conectividade do grafo aleatório ErdösRényi, e uma extensão deste modelo adicionando uma topologia simples, com conexões locais. Para isto foi preciso estudar o número de vértices isolados, tanto para o modelo Erdös-Rényi, como para a extensão deste modelo já mencionada.

Esta dissertação tem duas partes principais. Na primeira, estudei o limiar de conectividade no modelo Erdös-Rényi, tal como apresentado no livro de Hofstad da referencia [1]. Na segunda parte, usando os mesmos métodos de prova que neste livro, obtive resultados importantes relacionados à conectividade de um grafo variante do modelo Erdös-Rényi (o mais importante o Teorema 3.1.1), na qual tem uma topologia em que é adicionada uma probabilidade $q$ de conexão com os vizinhos mais próximos. O único resultado que não foi alcançado foi a condição para conseguir conectividade, o análogo à Proposição 2.2.2. O problema foi que não conseguimos estender o argumento envolvendo árvores. Provar este resultado é uma das possíveis continuações deste trabalho. Uma outra possibilidade seria considerar uma estrutura de vizinhança mais complexa, com alcance maior, autorizando que vértices a distância $k$ sejam conectados com probabilidade $q_{k}$. 


\section{Apêndice}

\section{A1. Permutações e Combinações}

\section{A1.1. Permutações simples}

O número de formas de ordenar $n$ objetos distintos é

$$
n !=n(n-1) \cdots 1
$$

Cada ordenação de $n$ objetos é chamada uma permutação simples de $n$ objetos. O número $n$ ! é chamado factorial de $n$. Por convenção $0 !=1$.

Observação: Uma fórmula muito importante quando se trata de factoriais foi obtida por Stirling (1730).

$$
n ! \sim n^{n} e^{-n} \sqrt{2 \pi n},
$$

onde o simbolo $\sim$ indica que a razão entre os dois lados tende a 1 quando $n \rightarrow \infty$.

\section{A1.2. Combinações simples}

De quantos modos podemos escolher $r$ objetos distintos entre $n$ objetos distintos dados?

Em geral, como $n(n-1) \cdots(n-r+1)$ representa o número de diferentes maneiras pelas quais um grupo de $r$ objetos pode ser selecionado a partir de $n$ objetos quando a ordem da seleção é relevante, e como cada grupo de $r$ objetos será contado $r$ ! vezes, tem-se que o número de grupos diferentes de $r$ objetos que podem ser formados a partir de um conjunto de $n$ objetos é

$$
\frac{n(n-1) \cdots(n-r+1)}{r !}=\frac{n !}{(n-r) ! r !}
$$

Notação: Definimos $\left(\begin{array}{l}n \\ r\end{array}\right)$, para $r \leq n$, como

$$
\left(\begin{array}{l}
n \\
r
\end{array}\right)=\frac{n !}{(n-r) ! r !}
$$

E dizemos que $\left(\begin{array}{l}n \\ r\end{array}\right)$ representa o número de combinações possíveis de $n$ objetos em grupos de $r$ elementos de cada vez. Por convenção, $0 !=1$, com isto, $\left(\begin{array}{l}n \\ 0\end{array}\right)=\left(\begin{array}{l}n \\ n\end{array}\right)=1$. além disso, assume-se que $\left(\begin{array}{c}n \\ i\end{array}\right)=0$ quando $i<0$ ou $i>n$. 
Assim, $\left(\begin{array}{l}n \\ r\end{array}\right)$ representa o número de grupos diferentes com $r$ elementos que podem ser selecionados de um conjunto de $n$ objetos quando a ordem da seleção não é considerada relevante.

\section{A1.3. Primeiro Lema de Kaplansky}

De quantos modos é possível formar um subconjunto com $p$ elementos de $\{1,2, \ldots, n\}$ no qual não haja números consecutivos? Por exemplo, para $n=6$ e $p=3$, podemos obter a partir de $\{1,2,3,4,5,6\}$ os seguintes subconjuntos com 3 elementos, nos quais não há elementos consecutivos:

$$
\{1,3,5\}, \quad\{1,3,6\}, \quad\{1,4,6\}, \quad\{2,4,6\} \text {. }
$$

Poderíamos obter este resultado sem a necessidade de enumerará-los exaustivamente. Ao formar um subconjunto, marcamos com o sinal + os elementos do conjunto que farão parte do subconjunto e com o sinal - os elementos que não farão parte do subconjunto. Assim $\{1,3,5\}$ seria representado por $+-+-+-;\{2,3,6\}$ (que não é um subconjunto válido pois 2 e 3 são consecutivos) seria marcado -++--+ .

Ora, para formar subconjuntos com 3 elementos de $\{1,2, \ldots, 6\}$ sem elementos consecutivos, devemos colocar 3 sinais + e 3 sinais - em fila, sem que haja dois sinais + consecutivos. Para fazer isso, colocamos os sinais - (1 modo), e colocamos os sinais + nos 4 espaços $\vee$ assinalados em

$$
\vee-V-V-V
$$

com no máximo um sinal por espaço. A resposta é então $1 \times\left(\begin{array}{l}4 \\ 3\end{array}\right)=4$. No caso geral temos $p$ sinais,$+ n-p$ sinais - para arrumar sem que haja dois sinais + consecutivos. Temos um modo de colocar os sinais - e $\left(\begin{array}{c}n-p+1 \\ p\end{array}\right)$ modos de colocar os sinais + .

Assim obtemos o Primeiro Lema de Kaplansky: O número de subconjuntos com $p$ elementos de $\{1, \ldots, n\}$ nos quais não há números consecutivos é

$$
f(n, p)=\left(\begin{array}{c}
n-p+1 \\
p
\end{array}\right)
$$

Podemos encontrar este Lema em [6, cap. 3, pag. 72].

\section{A1.4. Segundo Lema de Kaplansky}

Suponhamos agora que os elementos de $\{1, \ldots, n\}$ estejam arrumados em círculo. Agora os elementos 1 e $n$ são consecutivos. De quantos modos é possível formar um subconjunto com $p$ elementos de $\{1, \ldots, n\}$ no qual não haja números consecutivos? Ora, o número total de subconjuntos será a soma do número de subconjuntos nos quais o elemento 1 figura, com o número de subconjuntos nos quais o elemento 1 não figura.

a) Subconjunto nos quais o elemento 1 figura. Para formá-los devemos escolher $p-1$ elementos em $\{2,3 \ldots, n-1\}$ (pois se o 1 figura, o 2 e o $n$ não podem figurar) para 
serem os companheiros do 1 no subconjunto, não podendo ser escolhidos elementos consecutivos. O número de modos de que isso pode ser feito é

$$
f(n-3, p-1)=\left(\begin{array}{c}
n-3-(p-1)+1 \\
p-1
\end{array}\right)=\left(\begin{array}{c}
n-p-1 \\
p-1
\end{array}\right)
$$

b) Subconjunto nos quais o elemento 1 não figura. Para formá-los devemos escolher $p$ elementos em $\{2,3 \ldots, n\}$, não podendo ser escolhidos elementos consecutivos. Isso pode ser feito de $f(n-1, p)=\left(\begin{array}{c}n-1-p+1 \\ p\end{array}\right)=\left(\begin{array}{c}n-p \\ p\end{array}\right)$ modos. Portanto, a resposta é

$$
\left(\begin{array}{c}
n-p-1 \\
p-1
\end{array}\right)+\left(\begin{array}{c}
n-p \\
p
\end{array}\right)=\frac{n}{n-p}\left(\begin{array}{c}
n-p \\
p
\end{array}\right)
$$

Assim obtemos o Segundo Lema de Kaplansky: O número de subconjuntos com $p$ elementos de $\{1, \ldots, n\}$ nos quais não há números consecutivos, considerando 1 e $n$ como consecutivos, é igual a

$$
g(n, p)=\frac{n}{n-p}\left(\begin{array}{c}
n-p \\
p
\end{array}\right)
$$

\section{A2. Probabilidades}

Uma função $\mathbb{P}$, é definida na $\sigma$-álgebra $\mathcal{F}$ de subconjuntos de $\Omega$ e com valores em $[0,1]$, é uma probabilidade se satisfaz os seguintes axiomas:

(1) $\mathbb{P}(\Omega)=1$;

(2) Para todo subconjunto $A \in \mathcal{F}, \mathbb{P}(A) \geq 0$;

(3) Para toda sequência $A_{1}, A_{2}, \ldots \in \mathcal{F}$, mutuamente exclusivos, temos

$$
\mathbb{P}\left(\sum_{i=1}^{\infty} A_{i}\right)=\sum_{i=1}^{\infty} \mathbb{P}\left(A_{i}\right) .
$$

A tripla $(\Omega, \mathcal{F}, \mathbb{P})$ é denominada espaço de probabilidade. Os subconjuntos em $\mathcal{F}$ são denominados eventos e é somente a eles que se atribui probabilidade.

\section{A2.1. Propriedades}

Dado um espaço de probabilidade $(\Omega, \mathcal{F}, \mathbb{P})$ considere que os conjuntos $A$ e $B$, são eventos nesse espaço de probabilidade. Temos

P1. Sendo $A$ e $B$ dois eventos quaisquer, vale

$$
\mathbb{P}(B)=\mathbb{P}(B \cap A)+\mathbb{P}\left(B \cap A^{c}\right)
$$

em que $A^{c}$ é o complementar de $A$. 
P2. Se $A \subset B$ então $\mathbb{P}(A) \leq \mathbb{P}(B)$.

P3. Para eventos quaisquer $A_{1}, A_{2}, \ldots$

$$
\mathbb{P}\left(\sum_{i=1}^{\infty} A_{i}\right) \leq \sum_{i=1}^{\infty} \mathbb{P}\left(A_{i}\right) .
$$

Esta última propriedade é conhecida como a Desigualdade de Boole.

\section{A3. Convergência de variáveis aleatórias}

Definição (Convergência de variáveis aleatórias)

(a) Uma sequência de variáveis aleatórias $X_{n}$ converge em distribuição à variável aleatória $X$, denotado por $X_{n} \stackrel{d}{\rightarrow} X$, quando

$$
\lim _{n \longrightarrow \infty} \mathbb{P}\left(X_{n} \leq x\right)=P(X \leq x),
$$

para todo $x$ tal que $F(x)=\mathbb{P}(X \leq x)$ é continua.

(b) Uma sequência de variáveis aleatórias $X_{n}$ converge em probabilidade à variável aleatória $X$, denotado por $X_{n} \stackrel{P}{\rightarrow} X$, quando, para todo $\varepsilon>0$

$$
\lim _{n \rightarrow \infty} \mathbb{P}\left(\left|X_{n}-X\right|>\varepsilon\right)=0 \text {. }
$$

(c) Uma sequência de variáveis aleatórias $X_{n}$ converge quase certamente à variável aleatória $X$, denotado por $X_{n} \stackrel{\text { a.s. }}{\rightarrow} X$, quando,

$$
\mathbb{P}\left(\lim _{n \longrightarrow \infty} X_{n}=X\right)=1 \text {. }
$$

Neste trabalho, vamos trabalhar principalmente com a convergência em distribuição e convergência em probabilidade. A relação entre estes tipos de convergência segue no seguinte teorema.

Teorema (Relação entre tipos de convergência.) A convergência em probabilidade implica a convergência em distribuição, isto é, se $X_{n} \stackrel{P}{\rightarrow} X$ então $X_{n} \stackrel{d}{\rightarrow} X$.

Prova. Sejam $F_{X_{n}}(x)$ e $F_{X}(x)$ as funções de distribuição acumulada das v.a's $X_{n}$ e $X$, respectivamente. Suponha que $X_{n} \stackrel{P}{\rightarrow} X$ e seja $x$ um ponto de continuidade de $F_{X}$.

Queremos provar que $F_{X_{n}}(x) \rightarrow F_{X}(x)$ quando $n \rightarrow \infty$. Para isto, seja $\varepsilon>0$, note que $X \leq X_{n}+\varepsilon$ ou $X>X_{n}+\varepsilon$. Logo, se $X_{n} \leq x$ então $X \leq x+\varepsilon$ ou $X-X_{n}=\left|X_{n}-X\right|>\varepsilon$. Logo sendo $\Omega$ o espaço amostral, temos que

$$
\left\{\omega \in \Omega: X_{n}(\omega) \leq x\right\} \subset\{\omega \in \Omega: X(\omega) \leq x+\varepsilon\} \cup\left\{\omega \in \Omega:\left|X_{n}-X\right|(\omega)>\varepsilon\right\} .
$$


Logo

$$
F_{X_{n}}(x)=P\left(X_{n} \leq x\right) \leq F_{X}(x+\varepsilon)+P\left(\left|X_{n}-X\right|>\varepsilon\right) .
$$

Por outro lado, para $\varepsilon>0$, note que $X_{n} \leq X+\varepsilon$ ou $X_{n}>X+\varepsilon$. Logo, se $X \leq x-\varepsilon$ então $X_{n} \leq x$ ou $X_{n}-X>\varepsilon$, de modo que

$$
F_{X}(x-\varepsilon) \leq F_{X_{n}}(x)+P\left(\left|X_{n}-X\right|>\varepsilon\right) .
$$

Juntando as duas desigualdades, temos $\forall \varepsilon>0, \forall n$,

$$
F_{X}(x-\varepsilon)-P\left(\left|X_{n}-X\right|>\varepsilon\right) \leq F_{X_{n}}(x) \leq F_{X}(x+\varepsilon)+P\left(\left|X_{n}-X\right|>\varepsilon\right) .
$$

Fazendo $n \rightarrow \infty$ e como $X_{n} \stackrel{P}{\rightarrow} X$

$$
F_{X}(x-\varepsilon) \leq \liminf _{n \rightarrow \infty} F_{X_{n}}(x) \leq \limsup _{n \rightarrow \infty} F_{X_{n}}(x) \leq F_{X}(x+\varepsilon) .
$$

Logo fazendo $\varepsilon \rightarrow 0$ e usando o fato de que $F_{X}$ é continua em $x$ concluímos que

$$
\lim _{n \rightarrow \infty} F_{X_{n}}(x)=F_{X}(x)
$$

ou seja $X_{n} \stackrel{d}{\rightarrow} X$. Também podemos encontrar a prova deste teorema em [5, cap. 6 , pag.219].

Exemplo 1: Seja a sequência de variáveis aleatórias $\left\{X_{n}\right\}_{n=1}^{\infty}$, tal que $P\left(X_{n}=n\right)=\frac{1}{n}$ e $P\left(X_{n}=0\right)=1-\frac{1}{n}$. Mostre que $X_{n}$ converge em distribuição e em probabilidade a 0 .

Temos que provar que para todo $\varepsilon>0, \lim _{n \rightarrow \infty} P\left(\left|X_{n}-X\right|>\varepsilon\right)=0$. Para isto, note que para todo $\varepsilon>0$, existe um $n^{\prime}$ tal que $\forall n>n^{\prime}$, então

$$
\mathbb{P}\left(\left|X_{n}\right|>\varepsilon\right)=\mathbb{P}\left(X_{n}=n\right)=\frac{1}{n} \rightarrow 0, \quad \text { quando } n \rightarrow \infty .
$$

Portanto $X_{n} \stackrel{P}{\rightarrow}$, e pelo Teorema de Relação entre tipos de convergência. podemos concluir que $X_{n} \stackrel{d}{\rightarrow} 0$. Isto é,

$$
\lim _{n \rightarrow \infty} P\left(X_{n} \leq x\right)=P(X \leq x)= \begin{cases}1, & \text { se } x \geq 0 \\ 0, & \text { se } x<0\end{cases}
$$

A implicação recíproca no Teorema de Relação entre tipos de convergência. não necessariamente é verdadeira, com o seguinte exemplo mostramos esta afirmação.

Exemplo 2: Seja $X \sim N(0,1)$, tome

$$
X_{n}=\left\{\begin{array}{cc}
-X & \text { para } n \text { par } \\
X & \text { para } n \text { impar }
\end{array}\right.
$$

Veja que $X_{n}$ também segue uma distribuição Normal padrão para todo $n$, então $X_{n} \stackrel{d}{\rightarrow} X$. Logo para $n$ par, temos

$$
\begin{aligned}
P\left(\left|X_{n}-X\right|>\varepsilon\right) & =P(|-X-X|>\varepsilon) \\
& =P(|X|>\varepsilon / 2) \\
& =P(X>\varepsilon / 2)+P(X<-\varepsilon / 2) \\
& =2 P(X>\varepsilon / 2)>0 .
\end{aligned}
$$


Então $P\left(\left|X_{n}-X\right|>\varepsilon\right) \neq 0$ para $\varepsilon>0$ tão pequeno como quiser, portanto $X_{n}$ não converge em probabilidade para $X$.

\section{A4. Definição das funções $o$ e $O$}

Muitas vezes, é importante falar sobre a taxa na qual alguma função muda quando seu argumento cresce (ou diminui), sem se preocupar muito com a forma detalhada. Isto é o que as notações $O(\cdot)$ e $o(\cdot)$ nos permitem fazer.

A função $f(n)$ é "de ordem constante", ou "de ordem 1" quando existe alguma constante $c$ não nula tal que $f(n) / c \rightarrow 1$ quando $n \rightarrow \infty$; equivalentemente, desde que $c$ é uma constante $f(n) \rightarrow c$ quando $n \rightarrow \infty$. Então escrevemos $f(n)=O(1)$ e dizemos que "A constante de proporcionalidade $c$ é absorvido por o grande $O(\cdot)$ ". As outras ordens são definidos de forma recursiva, isto é,

$$
g(n)=O(f(n)) \text { se } \frac{g(n)}{f(n)}=O(1) \text { ou } \frac{g(n)}{f(n)} \rightarrow c \text { quando } n \rightarrow \infty
$$

é dizer, $g(n)$ é da mesma ordem que $f(n)$, e elas "crescem na mesma taxa".

O correspondente $o(\cdot)$ significa "é em última instancia menor do que": Dizemos que $f(n)=o(1)$ se $f(n) / c \rightarrow 0$ para alguma constante $c$. Recursivamente,

$$
g(n)=o(f(n)) \text { se } \frac{g(n)}{f(n)}=o(1) \text { ou } \frac{g(n)}{f(n)} \rightarrow 0 .
$$

Também lemos $g(n)=o(f(n))$ como " $g(n)$ é em última instância insignificante em comparação com $f(n)$ ".

\section{A5. Árvores}

\section{A5.1. Árvores}

Na teoria dos grafos, uma árvore é um grafo conexo (existe caminho entre quaisquer dois de seus vértices) e acíclico (não possui ciclos). Um ciclo em teoria de grafos é "um passeio de comprimento mínimo três, em que o primeiro e o último vértice coincidem, mas nenhum outro vértice é repetido". Caso o grafo seja acíclico mas não conexo, ele é dito uma floresta. Uma floresta também é definida como uma união disjunta de árvores.

Toda árvore é um grafo, mas nem todo grafo é uma árvore. Todo grafo conexo possui pelo menos uma árvore de extensão associada, composta de todos os seus vértices e algumas de suas arestas.

\section{Propriedades:}

Seja $G$ um grafo. $G$ é uma árvore se satisfaz as seguintes condições: 
1. G é conexo e há exatamente um caminho entre dois vértices quaisquer. Já em uma floresta, há no máximo um caminho entre dois vértices, devido à não conectividade.

2. $G$ é acíclico, e um simples ciclo é formado se qualquer aresta for adicionada a $G$.

3. $G$ é conexo, e deixará de ser conexo se qualquer aresta for removida de $G$.

4. $G$ é conexo, acíclico e tem $n-1$ arestas.

Proposição Um grafo $G$ é uma árvore se e somente se existir um único caminho entre cada par de vértices de $G$.

Definição (Árvore enraizada). Uma árvore é denominada enraizada se um vértice é escolhido como especial. Esse vértice é chamado raiz. Uma árvore que não é enraizada é denominada livre. O leitor pode encontrar os conceitos desde tema, definidos formalmente em $[10,11]$.

\section{A5.2. Árvore de extensão}

Uma árvore de extensão ou árvore de dispersão (em inglês: spanning tree) é o subconjunto de arestas de um grafo que forma uma árvore contendo todos os vértices. Uma árvore de extensão define um subconjunto de arestas que mantém o grafo conectado em um único componente.

Teorema de Cayley. O número de árvores etiquetados (marcados) de tamanho $n$ é igual a $n^{n-2}$. Equivalentemente, o número de árvores de extensão do grafo completo de tamanho $n$, é igual a $n^{n-2}$.

A prova deste Teorema pode-se encontrar em [1, cap. 3, pág. 75], ou também em [11, cap. 2, pág. 82].

\section{A6. Processos de Ramificação}

Um processo de ramificação é o modelo mais simples possível para uma população em evolução no tempo. Denotamos por $Z_{n} \operatorname{com} n \geq 0$ o número de indivíduos na $n$-ésima geração. Chamamos de raiz a $Z_{0}$ que representa o número de indivíduos no começo do processo, onde, por convenção $Z_{0}=1$. O espaço de estados $I$ de $Z_{n}$ é o conjunto dos inteiros positivos (incluindo 0). Supomos que cada partícula dá origem a $X$ partículas na próxima geração, que podemos chamar de descendentes, onde $X$ é uma variável aleatória positiva com distribuição $\left(p_{k}\right)_{k \geq 0}$. Ou seja,

$$
\mathbb{P}(X=k)=\mathbb{P}(O \text { individuo tenha } k \text { filhos })=p_{k} \text { para } k=0,1,2, \ldots
$$

Além disso, assumimos que o número de descendentes das várias partículas nas várias gerações são tomadas independentemente de acordo com a distribuição $\left(p_{k}\right)_{k \geq 0}$. Com 
todas estas pressuposições, vemos que $Z_{n}$ é um processo de Markov. Em particular, a probabilidade de transição de uma etapa é dado por

$$
p(i, j)=\mathbb{P}\left(Z_{n+1}=j \mid Z_{n}=i\right)=\mathbb{P}\left(\sum_{k=1}^{i} X_{k}=j\right) \quad \text { para } i \geq 1, j \geq 0,
$$

onde $\left(X_{k}\right)_{1 \leq k \leq i}$ é uma sequência de variáveis aleatórias i.i.d. com distribuição $p_{k}$. Assumimos que o estado 0 é um estado de absorção para $Z_{n}$, isto é,

$$
p(0, i)=0 \text { e } p(0,0)=1
$$

também assumimos que o primeiro momento para a distribuição de descendência existe e é dado por.

$$
\mathbb{E}[X]=\mu=\sum_{k=0}^{\infty} k p_{k}
$$

Definimos $\eta$ de forma que $\mathbb{P}\left(\right.$ extinção $\left.\mid Z_{0}=1\right)=\eta$. Se condicionarmos o número de partículas geradas na primeira geração teremos:

$$
\mathbb{P}\left(\text { extinção } \mid Z_{0}=1\right)=\sum_{j \geq 0} \mathbb{P}(\text { extinção } \mid X=j) \mathbb{P}(X=j) \text {. }
$$

Supondo que $Z=j$ a extinção irá ocorrer se cada partícula da descendência $j$ se extinguir. Por propriedade da cadeia de Markov a probabilidade de extinção para cada um desses $j$ é $\eta$. Além disso, esses processos $j$ são mutuamente independentes. Portanto, $\mathbb{P}($ extinção $\mid X=j)=\eta^{j}$. Logo, $\eta$ tem de ser a solução de

$$
\eta=\sum_{j \geq 0} p_{j} \eta^{j}
$$

Observe que $\eta=1$ é sempre solução desta equação. Quando que existe solução em $[0,1)$ ? A função geradora de probabilidades em relação à distribuição de descendência é dada por

$$
G_{X}(s)=\mathbb{E}\left[s^{X}\right]=\sum_{k=0}^{\infty} p_{k} s^{k} \quad \text { para }|s|<1 .
$$

Proposição. Seja $G_{1}=G$ e $G_{n+1}=G \circ G_{n}$ para $n \geq 1$. Para $n \geq 1$ a função geradora de $Z_{n}$ condicionado em $Z_{0}=1$ é $G_{n}$.

Prova. Vamos provar por indução. Seja $g_{n}$ a função geradora de $Z_{n}$ dado $Z_{0}=1$. Temos

$$
g_{1}(s)=\mathbb{E}\left[s^{Z_{1}} \mid Z_{0}=1\right]=\mathbb{E}\left[s^{X_{1}}\right]=G_{X}(s)=G_{1}(s) .
$$

Então vale para $n=1$. Suponha que $g_{n}=G_{n}$. Desde que, dado $Z_{n}=k$, a distribuição de $Z_{n+1}$ tem a mesma distribuição de $\sum_{i=1}^{k} X_{i}$ e os $X_{i}$ são v.a. iid. com distribuição $p_{k}$. Então

$$
\mathbb{E}_{1}\left[s^{Z_{n+1}} \mid Z_{n}=k\right]=\mathbb{E}_{1}\left[s^{\sum_{i=1}^{k} X_{i}}\right]=\left(\mathbb{E}\left[s^{X_{1}}\right]\right)^{k}=G_{X}(s)^{k},
$$




$$
g_{n+1}(s)=\mathbb{E}_{1}\left[s^{Z_{n+1}}\right]=\sum_{k=0}^{\infty} \mathbb{E}_{1}\left[s^{Z_{n+1}} \mid Z_{n}=k\right] \mathbb{P}_{1}\left(Z_{n}=k\right)
$$

Portanto

$$
g_{n+1}=\sum_{k=0}^{\infty} G_{X}(s)^{k} \mathbb{P}\left(Z_{n}=k \mid Z_{0}=1\right)=g_{n}\left(G_{X}(s)\right),
$$

pela hipótese de indução, $g_{n+1}=g_{n} \circ G=G_{n} \circ G=G_{n+1}$.

Proposição. Temos que $\mathbb{E}\left[Z_{n} \mid Z_{0}=1\right]=\mathbb{E}[X]^{n}=\mu^{n}$ para $n \geq 0$.

Prova. Recordemos que $\mathbb{E}\left[Z_{n}\right]=\mathbb{E}\left[\mathbb{E}\left[Z_{n} \mid Z_{n-1}\right]\right]$. Então

$$
\mathbb{E}\left[Z_{n}\right]=\mathbb{E}\left[Z_{n} \mid Z_{0}=1\right]=\sum_{k \geq 0} \mathbb{E}\left[Z_{n} \mid Z_{n-1}=k\right] \mathbb{P}\left(Z_{n-1}=k \mid Z_{0}=1\right),
$$

logo para $k \geq 1$, temos que

$$
\mathbb{E}\left[Z_{n} \mid Z_{n-1}=k\right]=\mathbb{E}\left[\sum_{i=1}^{k} X_{i}\right]=k \mathbb{E}[X]=k \mu,
$$

assim

$$
\mathbb{E}\left[Z_{n} \mid Z_{0}=1\right]=\sum_{k \geq 0} k \mathbb{E}[X] \mathbb{P}\left(Z_{n-1}=k \mid Z_{0}=1\right)=\mu \mathbb{E}\left[Z_{n-1} \mid Z_{0}=1\right],
$$

portanto, usando $\mathbb{E}\left[Z_{1} \mid Z_{0}=1\right]=\mu, \mathbb{E}\left[Z_{n} \mid Z_{0}=1\right]=\mu^{n}$.

Um dos principais resultados dos processos de ramificação é que quando $\mathbb{E}[X] \leq 1$, a população morre com probabilidade 1 , enquanto se $\mathbb{E}[X]>1$, há uma probabilidade não nula de que a população não irá extinguir-se. Denotamos a probabilidade de extinção por

$$
\eta=\mathbb{P}\left(\exists n: Z_{n}=0\right)
$$

Theorem. Suponha que $p_{0}+p_{1}<1$. O processo de ramificação exibe uma transição de fase no sentido de que

i. Se $\mathbb{E}[X] \leq 1$ então $\eta=1$

ii. Se $\mathbb{E}[X]>1$ então $\eta<1$

além disso $\eta$, a probabilidade de extinção, é a menor solução em $(0,1)$ da equação $G_{X}(\eta)=$ $\eta$ quando $\mathbb{E}[X]>1$.

Dizemos processo de ramificação subcrítico, quando $\mathbb{E}[X]<1$, e supercrítico quando $\mathbb{E}[X]>1$. Denotemos por $\zeta=1-\eta$ a probabilidade de sobrevivência, que é a probabilidade de que um processo sobreviva sempre, isto é, $\zeta=\mathbb{P}\left[Z_{n}>0 \forall n \geq 0\right]$, onde $\eta$ é a probabilidade de extinção. 


\section{A6.1. Processo de ramificação Poisson}

Um processo de ramificação Poisson é um processo com distribuição de descendência Poisson, denotamos esta distribuição por

$$
\mathbb{P}_{\lambda}^{*}=\mathbb{P}\left[X^{*}=x\right]=e^{-\lambda} \frac{\lambda^{x}}{x !},
$$

para uma v.a. $X^{*}$ Poisson e média de descendência $\lambda$. A função geradora de probabilidade da distribuição de descendência é igual a

$$
G *_{\lambda}(s)=\mathbb{E} *_{\lambda}\left[s^{X^{*}}\right]=\sum_{i=0}^{\infty} s^{i} e^{-\lambda} \frac{\lambda^{i}}{i !}=e^{\lambda(s-1)},
$$

portanto

$$
\eta_{\lambda}=G_{\eta_{\lambda}}(s)=e^{\lambda\left(\eta_{\lambda}-1\right)} .
$$

Para $\lambda \leq 1$, a equação tem uma única solução $\eta_{\lambda}=1$, que corresponde a uma extinção quase certa. Para $\lambda>1$, existem duas soluções, das quais a menor satisfaz $\eta_{\lambda} \in(0,1)$. Na figura 1, podemos ver o comportamento da probabilidade de sobrevivência $\zeta=\zeta_{\lambda}$, em função de $\lambda$, de um processo de ramificação com distribuição de descendência Poisson, com parâmetro $\lambda$.

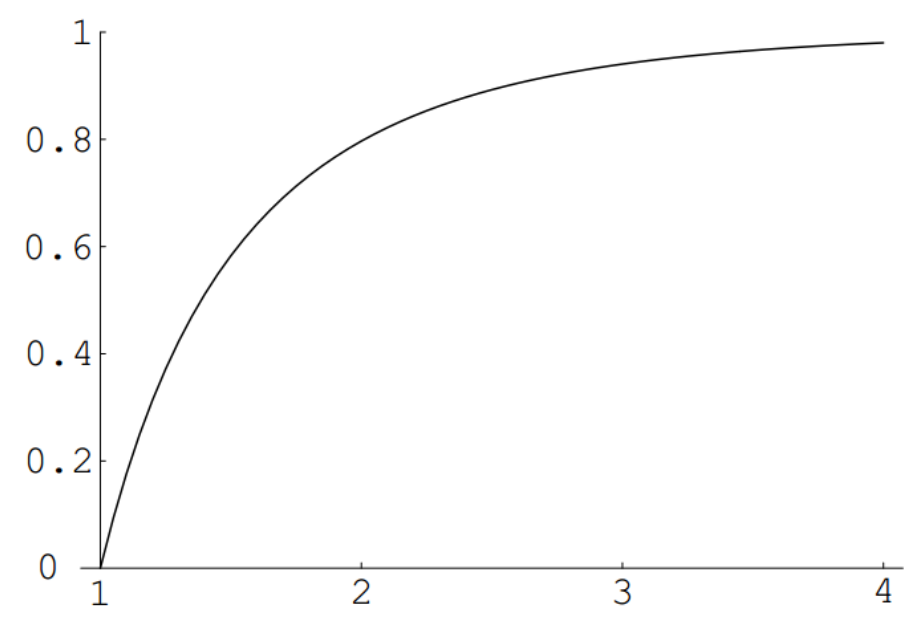

Figura 1: Probabilidade de sobrevivência, para um processo de ramificação com média $\lambda$. 


\section{Referências Bibliográficas}

[1] Remco Van Der Hofstad, Random Graphs and Complex Networks, Vol. I, Eindhoven University of Technology, The Netherlands, (2014).

[2] BÉla Bollobás, Random Graphs, volume 73 of Cambridge Studies in Advanced Mathematics. Cambridge University Press, Cambridge, second edition, (2001).

[3] W. Feller., An Introduction to Probability Theory and Its Applications, Volume I Wiley, New York, 3rd edition, 1968.

[4] Sheldon Ross, Probabilidade um curso modeno com aplicações, 8.ed. Univérsity of Southem California, 1968.

[5] Barry R. James, Probabilidade: Um Curso em Nivel Intermédio, IMPA, Rio de Janeiro, 3rd edition, 2013.

[6] Augusto C.O. Morgado, João B. P. De Carvalho, Paulo C. P. Carvalho, Pedro F., Analise Combinatório e Probabilidades Sociedade Brasileira de Matemática 2000.

[7] Newman, M. E., Watts, D. J., Strogatz, S. H.2002, Random Graph Model of Social Network Proceedings of the National Academy of Sciences, 99(suppl 1), 2566-2572.

[8] P. Erdös, A. RÉnyi, On Random Graph I Math. Debrecen, 6 1959, pp. 290-297.

[9] P. ERdös, A. RÉNyi, On Evolution of Random Graph On the evolution of random graphs, Institute of Mathematics Hungarian Academy of Sciences, Hungary, 5 1960, pp. 17-61.

[10] BÉla Bollobás, Modern Graph Theory. Graduate Texts in Mathematics 184 Springer Verlag, Neuw York 1988.

[11] D. B. West, Introduction to Graph Theory. Prentice Hall, New Jersey 1996.

[12] Botelho Ribeiro, R., Grafos aleatórios e Percolação. Mestrado em Matemática. Universidade Federal de Minas Gerais, 2012. 